\title{
Auto-Regressive Model Based Polarimetric Adaptive Detection Scheme Part II: Performance Assessment under Spectral Model Mismatch
}

\author{
Fabiola Colone, Senior Member, IEEE, and Francesca Filippini, Member, IEEE
}

\begin{abstract}
This work addresses the problem of target detection in coherent radar systems equipped with multiple polarimetric channels. In "Part I" of this two-part study, a multichannel auto-regressive model based polarimetric detection scheme has been developed and its performance has been studied against clutter with characteristics exactly matching the adopted parametric model. In this second part of the study, the performance assessment is extended, by means of theoretical and simulated analyses, to include the case of disturbance components with diverse spectral characteristics. Consequently, an appropriate modification is introduced to the detection scheme to make it robust to typical spectral mismatches occurring in practical situations. Finally, the effectiveness of the resulting detection scheme is proved against simulated and experimental data.
\end{abstract}

Index Terms - polarimetric radar, adaptive signal detection, parametric approach, multi-channel auto-regressive process

\section{INTRODUCTION}

$\mathrm{T}$ HIS work is divided into two parts [1] and is concerned with the problem of disturbance cancellation and target detection in coherent polarimetric radar systems. Traditional approaches to this problem are fully adaptive polarimetric detectors, such as the polarimetric generalized likelihood ratio test (GLRT) in [2]-[3] and the polarimetric adaptive matched filter (AMF) in [4]. Such methods adaptively exploit all the available degrees of freedom to perform disturbance cancellation in both the polarimetric and temporal domains. While being theoretically optimum under the assumption of a priori known disturbance characteristics, these approaches might be computationally intensive and might suffer of significant adaptivity loss in practical cases, especially when a limited number of training data is available.

To overcome these issues, in the companion paper [1], we exploit a parametric method to develop a new adaptive polarimetric detector by modeling the disturbance as a multichannel auto-regressive (AR) process [5]-[11]. For the readers' benefit, we summarize structures and characteristics

F. Colone and F. Filippini are with the Dept. of Information Engineering, Electronics and Telecommunications (DIET), Sapienza University of Rome, Rome 00184, Italy, (e-mail: fabiola.colone@ uniroma1.it,

francesca.filippini@uniroma1.it) (Corresponding author: F. Colone) of the proposed AR model based polarimetric detection scheme in Section II of this paper.

However, the reader is advised to refer to [1] and the references therein to gain a deep understanding of the algorithm and its analysis. Specifically, a throughout performance analysis is reported in [1] for the case of an input disturbance process that exactly matches the AR model exploited for the design of the proposed detection scheme. In such conditions, the proposed detector has been shown to provide remarkable advantages over the traditional fullyadaptive approaches in terms of both target detection capability and computational complexity.

In this second part of the study, the performance analysis of the proposed polarimetric detector is extended to include the more general case of spectral model mismatches, namely the case of input disturbance with spectral characteristics differing from the AR model adopted to design the detector. We observe here that this is a typical condition in real-world applications where no a priori information is available on the disturbance impairing the received data, which in turn could result from the combination of multiple interference sources (e.g. clutter, noise, jammers) with quite different spectral characteristics. Moreover, despite effective approaches could be exploited to identify a suitable AR model that approximates the actual disturbance characteristics [12], the resulting approximation might not be perfect thus yielding a residual spectral model mismatch. Therefore, it is of high practical interest to investigate the effect of such mismatches on the performance of the proposed detector, namely the price to be paid for having resorted to a parametric method.

To this purpose, we first provide theoretical expressions for the asymptotic detection performance achievable with the detector proposed in [1], for a generic spectral mismatch encoded in the disturbance covariance matrix. Then, these results are compared to numerical analyses performed for two different types of spectral mismatches, i.e. mismatches in the order for the adopted AR model and mismatches in the considered spectral model (a Gaussian power spectral density is considered in this latter case).

The performed analysis allows to clearly identify the limitations of the polarimetric detector proposed in [1] when operated under mismatched conditions and this leads to the design of proper modifications to make it robust to limited 
spectral mismatches. Specifically, a modified doubly adaptive scheme is proposed able to guarantee a good control of the false alarm rate in practical situations and to contain the detection loss. The addition of a further adaptive processing stage is shown to yield increased adaptivity loss with respect to the original detection scheme in [1] since this loss is traded for a reduced mismatch loss. Overall, the modified AR based polarimetric adaptive detector is demonstrated to maintain an advantage in term of target detection capability over the traditional fully adaptive approaches [2]-[4] in the presence of limited spectral model mismatches for the disturbance. Also, this advantage comes with a significantly reduced computational complexity.

These conclusions have been further confirmed by means of application of the proposed detection scheme to different sets of experimental polarimetric radar data. The reported results clearly show that the modified AR based polarimetric adaptive detector represents an effective and reliable solution in practical applications.

The remainder of this manuscript is organized as follows. In Section II we briefly recall the AR model based polarimetric detector introduced in [1] and provide theoretical expressions for its detection performance under spectral model mismatches. A numerical analysis is then reported in Section III for two different case studies. The modified AR model based polarimetric adaptive detector is presented in Section IV whereas its performance is assessed in Sections V and VI against simulated and real data, respectively. Eventually, we draw our conclusions in Section VII.

\section{Theoretical ASYMPtotic Performance UNDER DISTURBANCE MODEL MISMATCH}

In the companion paper [1], the polarimetric detector PolAR-MF has been derived for the case of a polarimetric radar system equipped with $L$ polarimetric channels and operating over coherent processing intervals of $M$ samples.

Specifically, by assuming the target component to be partially structured (i.e. its returns are assumed to be known up to an unknown amplitude in the temporal domain but they involve unknown nonlinear signal parameters in the polarimetric domain) and by modeling the disturbance as an $L$-channel AR process with order $Q-1$ and known coefficients matrices $\mathbf{A}(L(Q-1) \times L)$ and $\mathbf{R}(L \times L)$, the clairvoyant Pol-AR-MF detector was obtained as

$$
\begin{gathered}
H_{1} \\
T_{P o l-A R-M F}=\mathbf{x}_{0}^{H} \mathbf{B ~ C ~ C ~}^{H} \mathbf{B}^{H} \mathbf{x}_{0} \gtrless \eta_{A R-M F} \\
H_{0}
\end{gathered}
$$

where $\mathbf{x}_{0}$ is the vector where we arrange the primary data samples collected at $M$ consecutive temporal observations from the $L$ available polarimetric channels. Matrices $\mathbf{B}$ and $\mathbf{C}$ depend on the $\operatorname{AR}(Q-1)$ parameters as well as on the target temporal steering vector; their definitions are detailed in [1] and are briefly summarized in Table I.
In the companion paper [1], we addressed the 'matched' case, namely the case of an input disturbance that exactly matches the $\operatorname{AR}(Q-1)$ model adopted for the design of the detection scheme and we provided analytical expressions of the asymptotic performance of the derived detector. More precisely, the $P_{f a}$ expression to be used when setting the detection threshold is given by eq. (29) of [1] and it is reported here for ease of reference

$$
P_{f a}=\sum_{l=0}^{L-1} \frac{\eta^{l}}{2^{l} \Gamma(L-l)} e^{-\frac{\eta}{2}}
$$

being $\eta$ the detection threshold, while eqs. (31) and (32) of [1] report the asymptotic detection probability $P_{d}$ expressions for the Swerling 0 and Swerling I target model [13]-[14], respectively.

In the present work, we address the general case of an input disturbance that is not drawn from the assumed $\operatorname{AR}(Q-1)$ model. Still, we assume that matrices $\mathbf{B}$ and $\mathbf{C}$ in (1) are evaluated based on the parameters of a multichannel AR process of given order $(Q-1)$. In other words, we evaluate matrices $\mathbf{A}_{m i s}(L(Q-1) \times L)$ and $\mathbf{R}_{m i s}(L \times L)$ starting from the actual data covariance matrix $\mathbf{M}$, i.e. $\mathbf{M}=E\left\{\mathbf{x}_{0} \mathbf{x}_{0}^{H}\right\}$, via the following relations

$$
\left\{\begin{array}{c}
\mathbf{A}_{m i s}=\overline{\mathbf{M}}_{00}^{-1} \overline{\mathbf{M}}_{01} \\
\mathbf{R}_{m i s}=\overline{\mathbf{M}}_{11}-\overline{\mathbf{M}}_{01}^{H} \overline{\mathbf{M}}_{00}^{-1} \overline{\mathbf{M}}_{01}
\end{array}\right.
$$

being $\overline{\mathbf{M}}=\left[\begin{array}{ll}\overline{\mathbf{M}}_{00} & \overline{\mathbf{M}}_{01} \\ \overline{\mathbf{M}}_{01}^{H} & \overline{\mathbf{M}}_{11}\end{array}\right]$ the first $Q L \times Q L$ block of matrix $\mathbf{M}$,

\begin{tabular}{|c|c|}
\hline Quantity & Definition/meaning \\
\hline B & {$\left[\begin{array}{llll}\mathbf{B}_{0} & \mathbf{B}_{1} & \ldots & \mathbf{B}_{M-Q}\end{array}\right]$} \\
\hline C & $\left(\mathbf{1}_{M-Q+1 \times 1} \otimes \mathbf{W}^{-\frac{1}{2}}\right)$ \\
\hline $\mathbf{B}_{m}$ & {$\left[\begin{array}{c}\mathbf{0}_{L m \times L} \\
\mathbf{P \Sigma}(m) \\
\mathbf{0}_{L(M-Q-m) \times L}\end{array}\right]$} \\
\hline w & $\frac{1}{2} \sum_{k=0} \boldsymbol{\Sigma}^{H}(m) \mathbf{P} \boldsymbol{\Sigma}(m)$ \\
\hline $\mathbf{P}$ & {$\left[\mathbf{H}^{H} \mathbf{R}^{-1} \mathbf{H}\right]$} \\
\hline H & {$\left[\begin{array}{ll}-\mathbf{A}^{H} & \mathbf{I}_{L}\end{array}\right]$} \\
\hline $\boldsymbol{\Sigma}(m)$ & $\tilde{\mathbf{t}}(m) \otimes \mathbf{I}_{L}$ \\
\hline $\mathbf{I}_{L}$ & $L \times L$ identity matrix \\
\hline$\tilde{\mathbf{t}}(m)$ & $\begin{array}{l}Q \text {-dimensional sub-vector of the temporal } \\
\text { steering vector starting from the } m \text {-th sample }\end{array}$ \\
\hline
\end{tabular}
with $\overline{\mathbf{M}}_{00}(L(Q-1) \times L(Q-1)), \overline{\mathbf{M}}_{01}(L(Q-1) \times L)$ and $\overline{\mathbf{M}}_{00}(L \times L)$.

Table I

SUMMARY OF DEFINED QUANTITIES 
As a consequence, matrices $\mathbf{A}_{\text {mis }}$ and $\mathbf{R}_{\text {mis }}$ represent the parameters of an AR model that possibly approximates the actual spectral characteristics of the disturbance but does not exactly match them. Therefore, the filtering of the data via matrices $\mathbf{B}$ and $\mathbf{C}$ does not provide a perfect whitening in either the polarimetric and the temporal domain. In contrast, some residual correlation might appear. These residuals in turn depend on the actual characteristics of the input disturbance and are expected to degrade the performance of the detector both in terms of $P_{f a}$ control and $P_{d}$. These effects are investigated in the following for the clairvoyant detector in (1). The reported analysis is also representative of the asymptotic performance, of the adaptive version of the proposed detector, the Pol-AR-AMF [1], under spectral model mismatch. Specifically, provided that a large number of training data $P$ is available, we assume that the ML estimate of the covariance matrix $\widehat{\mathbf{M}}$ tends towards the actual disturbance covariance matrix $\mathbf{M}$ and, consequently, the estimated AR parameters tend towards the clairvoyant, though mismatched, values in (3).

In sub-section II-A, we focus on the probability of false alarm while corresponding expressions for the target detection probability are developed in sub-section II-B.

\section{A. Probability of false alarm}

Let us consider the test statistic of the clairvoyant detector in (1) and define the $L$-dimensional vector $\breve{\mathbf{z}}_{0}=\mathbf{C}^{H} \mathbf{B}^{H} \mathbf{x}_{0}$, namely $\breve{\mathbf{z}}_{0}$ represents the data after both polarimetric and temporal disturbance cancellation, which then undergoes the non-coherent integration across the polarimetric channels.

Under the $H_{0}$ hypothesis, $\breve{\mathbf{z}}_{0}$ is a zero-mean complex Gaussian random vector with covariance matrix $\mathbf{D}_{0}=$ $\mathbf{C}^{H} \mathbf{B}^{H} \mathbf{M} \mathbf{B C}$, being $\mathbf{M}$ the actual covariance matrix of the input disturbance process, i.e. $\left.\breve{\mathbf{z}}_{0}\right|_{H_{0}} \sim \mathcal{C N}\left(\mathbf{0}_{L \times 1}, \mathbf{D}_{0}\right)$.

Depending on the form taken by the matrix $\mathbf{M}$, the test statistic in (1), namely $T_{P o l-A R-M F}=\left\|\breve{\mathbf{z}}_{0}\right\|^{2}$ might have different distributions.

For the 'matched' case, we recall that $\mathbf{D}_{0}=2 \mathbf{I}_{L}$ (see Appendix C of the companion paper [1] for proof). In contrast, for the general case when the input disturbance process does not strictly follow the $\operatorname{AR}(Q-1)$ model, some residual correlation might appear after the temporal cancellation stage so that $\mathbf{D}_{0} \neq 2 \mathbf{I}_{L}$ and (2) is no longer valid.

However, we can resort to the same approach reported in Appendix D of the companion paper to derive a closed form expression for the $P_{f a}$. Let $\lambda_{0}, \ldots, \lambda_{N-1}$ denote the $N \leq L$ distinct non-zero eigenvalues of $\mathbf{D}_{0}$, each with multiplicity $\mu_{n}, n=0, \ldots, N-1$. By proceeding as in [16], we obtain the $P_{f a}$ expression as follows

$$
P_{f a}=\sum_{n=0}^{N-1} \sum_{k=0}^{\mu_{n}-1} \frac{-e^{-\frac{\eta}{\lambda_{n}}} \eta^{k}}{\Gamma(k+1)} \delta_{k, n}
$$

where $\eta$ is the threshold, and the definition of the coefficients $\delta_{k, n}\left(k=0, \ldots, \mu_{n}-1, n=0, \ldots, N-1\right)$, is detailed in the companion paper [1] (see eq. (56) of Appendix D). By inverting (4) the threshold $\eta_{A R-M F}$ can be obtained for the clairvoyant detector in (1).

In the special case when no eigenvalue is repeated, which is typically verified in practical cases under spectral mismatch, namely when $N=L$ and $\mu_{n}=1, n=0, \ldots, N-1$, eq. (4) can be simplified and the following $P_{f a}$ is easily obtained

$$
P_{f a}=\sum_{l=0}^{L-1} \frac{\lambda_{l}^{L-1}}{\prod_{\substack{i=0 \\ i \neq l}}^{L-1}\left(\lambda_{l}-\lambda_{i}\right)} \mathrm{e}^{-\frac{\eta}{\lambda_{l}}}
$$

In the dual special case when matrix $\mathbf{D}_{0}$ has one unique eigenvalue $\lambda_{0}(N=1)$ with multiplicity $\mu_{0}=L$, the test statistic turns into a Gamma distributed variable, i.e.

$$
T_{\text {Pol-AR-MF }} \sim \Gamma\left(L, \lambda_{0}\right)
$$

and the $P_{f a}$ expression can be simplified as

$$
P_{f a}=\sum_{k=0}^{L-1} \frac{\left({ }^{\eta} / \lambda_{0}\right)^{k} e^{-\frac{\eta}{\lambda_{0}}}}{\Gamma(k+1)}
$$

Note that (7) is equivalent to (2), if the scale parameter $\lambda_{0}=$ 2 , namely if a perfect whitening has been obtained in both the polarimetric and temporal domain. In the general case, we expect that the higher similarity exists between the adopted AR model and the actual correlation characteristics of the disturbance process, the better this is rejected and the closer (4) gets to (2).

In contrast, in the general spectral mismatched case, the derived $P_{f a}$ expressions, and hence the threshold $\eta_{A R-M F}$ to be used, depend on the eigenvalues of the residual disturbance matrix $\mathbf{D}_{0}$ that are unknown in practical cases. Therefore, by recalling that the reported performance is representative of the performance of the adaptive polarimetric detector Pol-ARAMF for large number of training data, we conclude that it does not ensure the CFAR property even in the asymptotic regime if spectral model mismatches exists. The CFAR characteristic tends to be guaranteed only if the adopted model reasonably approximates the spectral characteristics of the disturbance.

\section{B. Probability of detection}

The disturbance residuals arising from the cancellation stage are also responsible of degradations in terms of target detection capability. These are theoretically investigated in the following with reference to a Swerling 0 target model and a Swerling I target model, respectively.

\section{1) Non-fluctuating target model (Swerling 0)}

When a non-fluctuating target model (Swerling 0) is assumed [13]-[14], vector $\breve{\mathbf{z}}_{0}$ is a complex Gaussian random vector, with mean vector $\mathbf{v}=\mathbf{C}^{H} \mathbf{B}^{H} \mathbf{S}$, being $\mathbf{s}$ the target component in the received primary data, and covariance matrix $\mathbf{D}_{0}$, i.e. $\left.\breve{\mathbf{z}}_{0}\right|_{H_{1}} \sim \mathcal{C N}\left(\mathbf{v}, \mathbf{D}_{0}\right)$. According to this model, the test statistic of the clairvoyant detector, $T_{P o l-A R-M F}=$ 
$\left\|\breve{\mathbf{z}}_{0}\right\|^{2}$, is a non-central quadratic form and the $P_{d}$ cannot be written in a closed form for any $\mathbf{D}_{0}$. However, following the approach in [16], in the Appendix we develop an approximated expression for the asymptotic $P_{d}$ that can be written as:

$$
\begin{aligned}
P_{d} \approx & 1-\frac{e^{-p_{0} \eta}}{p_{0} \sqrt{2 \pi}} \sum_{l=0}^{L-1} \frac{1}{1-p_{0} \lambda_{l}} \\
& \times \frac{e^{\sum_{l=0}^{L-1}\left|\overline{\bar{v}}_{l}\right|^{2}\left[\frac{1}{1-p_{0} \lambda_{l}}-1\right]}}{\sqrt{\left|\frac{1}{p_{0}^{2}}-\sum_{l=0}^{L-1}\left[\frac{\lambda_{l}{ }^{2}}{\left(1-p_{0} \lambda_{l}\right)^{2}}\left(1+\frac{2\left|\overline{\bar{v}}_{l}\right|^{2}}{1-p_{0} \lambda_{l}}\right)\right]\right|}}
\end{aligned}
$$

where $\eta$ is the detection threshold evaluated from (4), $p_{0}=$ $-\left(j \omega_{0}+\beta\right)$ and the definitions of $\omega_{0}$ and $\beta$ are detailed in the Appendix.

\section{2) Fluctuating target model (Swerling 1)}

Assuming a Swerling I model for the target [13]-[14], namely if the target complex amplitude is a zero-mean Gaussian random variable with covariance matrix $\mathbf{M}_{t}=$ $E\left\{\boldsymbol{\alpha} \boldsymbol{\alpha}^{H}\right\}$, vector $\breve{\mathbf{z}}_{0}$ is a complex Gaussian random variable with zero-mean vector and covariance matrix $\mathbf{D}_{0}^{\prime}=\mathbf{D}_{0}+$ $\mathbf{C}^{H} \mathbf{B}^{H}\left(\mathbf{t t}^{H} \otimes \mathbf{M}_{\mathrm{t}}\right) \mathbf{B C}$, i.e. $\left.\breve{\mathbf{z}}_{0}\right|_{H_{1}} \sim \mathcal{C} \mathcal{N}\left(\mathbf{0}_{L \times 1}, \mathbf{D}_{0}^{\prime}\right)$.

Therefore, following the same procedure as for the $P_{f a}$ in sub-section II-A, the $P_{d}$ is obtained as

$$
P_{d}=\sum_{n=0}^{N-1} \sum_{k=0}^{\mu_{n}^{\prime}-1} \frac{-e^{\left(-\frac{\eta}{\lambda_{n}^{\prime}}\right)} \eta^{k}}{\Gamma(k+1)} \delta_{k, n}^{\prime}
$$

where $\eta$ is the detection threshold, $\lambda_{0}^{\prime}, \ldots, \lambda_{N-1}^{\prime}$ denote the $N \leq L$ distinct eigenvalues of $\mathbf{D}_{0}^{\prime}$, each with multiplicity $\mu_{n}^{\prime}$, and the coefficients $\delta_{k, n}^{\prime}$ are evaluated using eq. (56) of Appendix D of the companion paper, by replacing $\lambda_{n}$ with $\lambda_{n}^{\prime}$.

\section{ASYMPTOTIC PERFORMANCE ANALYSIS AGAINST SIMULATED DATA}

In this Section, we carry out an asymptotic performance analysis of the proposed detector via numerical examples. The purpose of this Section is twofold. First, we aim at verifying the validity of the theoretical performance expressions, then we aim at investigating the performance loss only due to the spectral model mismatch, neglecting the additional degradation introduced by the adaptivity that will be considered later in this paper.

To this aim, we carried on extensive Monte Carlo (MC) simulations in two different case-studies, as detailed in the following.

- Case study A. In the first case study we assume that the disturbance is a $L$-channel AR (3) process and we investigate the robustness of the proposed detector when a model order mismatch occurs, namely when the detector is build using $Q \neq 4$. Specifically, the disturbance affecting the system is generated using the same parameters adopted in [1] with $L=3(\mathrm{HH}, \mathrm{VV}, \mathrm{HV})$ polarimetric channels and $M=32$.

- Case study B. In the second case study we consider the model used in [3], where a Gaussian spectral shape is adopted for the disturbance, and we investigate the robustness of the proposed detector that is based on a multichannel AR model approximation. To this purpose, we generate a disturbance characterized by a disturbance covariance matrix $\mathbf{M}$ that can be written as $\mathbf{M}=\boldsymbol{\Pi} \otimes \mathbf{Y}+$ $\sigma_{n}^{2} \mathbf{I}_{L M}$, denoting $\boldsymbol{\Pi}$ as the normalized temporal covariance matrix shared by all polarimetric channels, $\mathbf{Y}$ as the disturbance polarimetric covariance matrix and $\sigma_{n}^{2}$ as the noise power, defined with respect to the clutter power at $\mathrm{HH}$ and VV channels $\sigma_{d}^{2}$ via the clutter-to-noise ratio (CNR) of 40dB. Specifically, we assume ח to be Gaussian shaped with one-lag correlation coefficient $\varrho=0.95$, namely the generic element $\Pi_{m, p}=\varrho^{(m-p)^{2}},(m, p=0, \ldots$, $M$ - 1). We consider $M=32$ and $L=3$ (HH,VV,HV), and we assume that the $\mathrm{HH}$ and VV channel share the same disturbance power level, i.e. $\sigma_{d, H H}^{2}=\sigma_{d, V V}^{2}=\sigma_{d}^{2}$, deliberately set to $\sigma_{d}^{2}=1$, while the $\mathrm{HV}$ channel is generated with power level $20 \mathrm{~dB}$ lower than in $\mathrm{HH}$ and $\mathrm{VV}$, i.e. $\sigma_{d, H V}^{2}=\xi_{d} \sigma_{d}^{2}$, with $\xi_{d}=0.01$. Furthermore, a correlation coefficient equal to $\rho_{H H / V V}=0.9$ is set between the $\mathrm{HH}$ and $\mathrm{VV}$ channels, while the cross-polarized components are assumed independent from the copolarized ones, i.e. $\rho_{H V / H H}=\rho_{H V / V V}=0$ thus resulting in null cross-spectra. The auto- and cross- spectra of the available polarimetric channels are reported in Fig. 1. Ultimately, the disturbance polarimetric covariance matrix can be written as follows

$$
\mathbf{Y}=\sigma_{d}^{2}\left[\begin{array}{ccc}
1 & \rho_{H H / V V} & 0 \\
\rho_{H H / V V} & 1 & 0 \\
0 & 0 & \xi_{d}
\end{array}\right]
$$

In Fig. 2(a), we plot the $P_{f a}$ versus threshold obtained in case study A when employing the detector in (1) with a grid of $Q$ values, encoded by different brown shades and line styles.

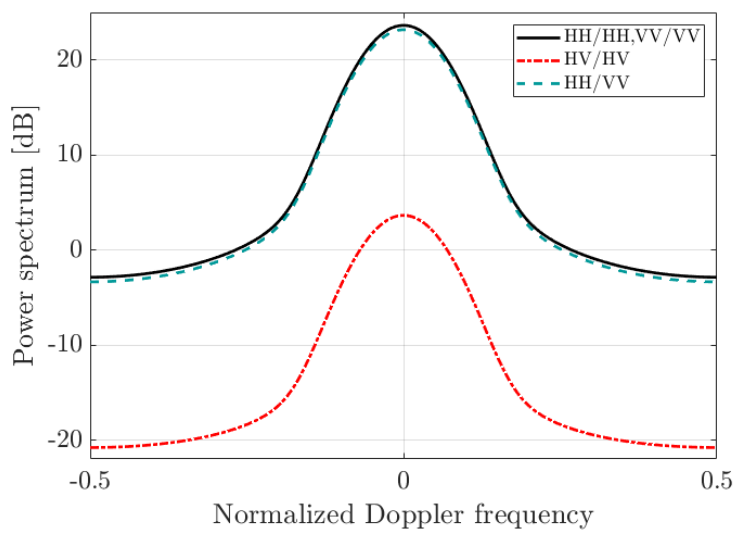

Fig. 1 Power spectra of case study B. 
Specifically, the reported curves have been obtained using the theoretical expression in (4), whereas the markers correspond to the results of MC simulations for the Pol-ARMF. On the same figure, we also report in green the $P_{f a}$ expression obtained from (2). This curve is representative of spectral 'matched' case, i.e. a perfect whitening is assumed for the disturbance in both temporal and polarization domains. Similarly, in Fig. 2(b) we report the results for case study B.

Fig. 2 (a-b) show that:

- if a spectral model mismatch occurs, the detection threshold must be properly adjusted to guarantee the nominal $P_{f a}$ and the required modification is largely dependent on the mismatch extent. This confirms that the CFAR property is not ensured even under the asymptotic condition.

- However, the higher the similarity between the AR process used to build the detection test and the true spectral characteristics of the disturbance, the closer gets the theoretical $P_{f a}$ expression to (2), revealing that a better disturbance cancellation was performed.

- Depending on the spectral characteristics of the input disturbance, the $Q$ value that allows to have acceptable mismatch loss typically changes. As is expected, when the detector is fed with an AR disturbance process of order $\bar{Q}-$ 1 (case study A, Fig. 2 (a)), the mismatch loss increases when both underestimating or overestimating the model order with respect to the exact value $Q=\bar{Q}=4$. In contrast, when case study B is considered (Fig. 2 (b)), as $Q$ increases, the brown curves tend to look alike and to resemble the green curve. For instance, in this case, $Q \geq 15$ should be adopted to achieve an acceptable approximation of the Gaussian shaped power spectral density based on a multichannel AR model, since a reasonably high temporal correlation coefficient was assumed in this case study.

For the performance evaluation under the $H_{1}$ hypothesis, both Swerling 0 and Swerling I target models are considered, in Fig. 3 and Fig. 4, respectively. The target normalized Doppler frequency was set to $f_{d}=0.25$ and the same set of parameters are adopted as in [1]. In particular, when a Swerling 0 target model is used (see Fig. 3), the deterministic target complex amplitudes vector is set as $\boldsymbol{\alpha}=$ $a_{t}\left[\begin{array}{lll}1 & \mathrm{e}^{j \Delta \phi_{H H / V V}} & \sqrt{\xi_{t}} \mathrm{e}^{j \Delta \phi_{H H / H V}}\end{array}\right]^{T}$, where $\xi_{t}=0.1$, $\Delta \phi_{H H / V V}=\pi / 4$, and $\Delta \phi_{H H / H V}=\pi / 2$.

When a fluctuating target model is adopted according to a Swerling I model (see Fig. 4), vector $\boldsymbol{\alpha}$ is generated as a zeromean Gaussian random vector, with covariance matrix

$$
\mathbf{M}_{t}=\sigma_{t}^{2}\left[\begin{array}{lll}
1 & 0 & 0 \\
0 & 1 & 0 \\
0 & 0 & \xi_{t}
\end{array}\right]
$$

where $\xi_{t}$ has been set to $\xi_{t}=0.1$.

The results are reported for the two case studies A (see Fig. 3 (a) and Fig. 4(a) ) and B (see Fig. 3 (b) and Fig. 4(b)) as a function of the signal-to-clutter ratio (SCR) at the first

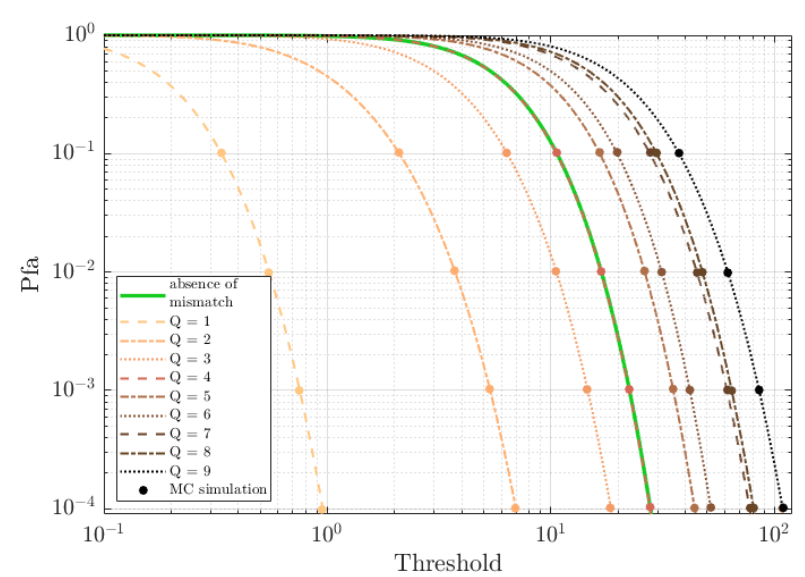

(a)

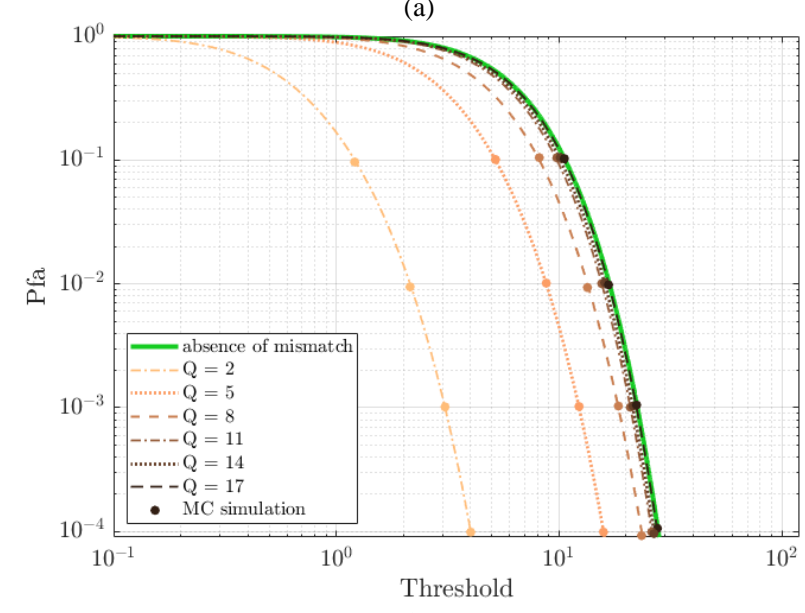

(b)

Fig. $2 P_{f a}$ versus threshold for different values of $Q$ for:

(a) case study A, (b) case study B.

polarimetric channel, i.e. $\mathrm{SCR}=\left|a_{t}\right|^{2} / \sigma_{d}^{2}$ in Fig. 3 and $\mathrm{SCR}=\sigma_{t}^{2} / \sigma_{d}^{2}$ in Fig. 4 . The $P_{f a}$ has been set to $10^{-3}$.

In all figures, we plot in dash-dot red the $P_{d}$ of the polarimetric matched filter (Pol-MF, [1]) and we consider it as a benchmark of our performance evaluation since this detector does not make any assumption on the spectral shape of the actual disturbance but is based on the exact knowledge of the input disturbance covariance matrix. Also, in each figure, we compare the MC simulation results of the Pol-ARMF with the appropriate theoretical expressions, i.e. from either (8) or (9), depending on the target model, for three different values of $Q$, namely $Q=2,4$ and 8 .

By observing Fig. 3 and Fig. 4, the following considerations apply.

- Both the closed-form and the approximate theoretical expressions match well with the results of the MC simulations, implying that the obtained $P_{d}$ expressions can accurately describe the asymptotic detection performance of the proposed detector under spectral mismatches. We recall that, in case study A, the detector operating with $Q=4$ represents the 'spectral matched' detector investigated in [1] . Consequently, in Fig. 2(a) also the exact expression derived 


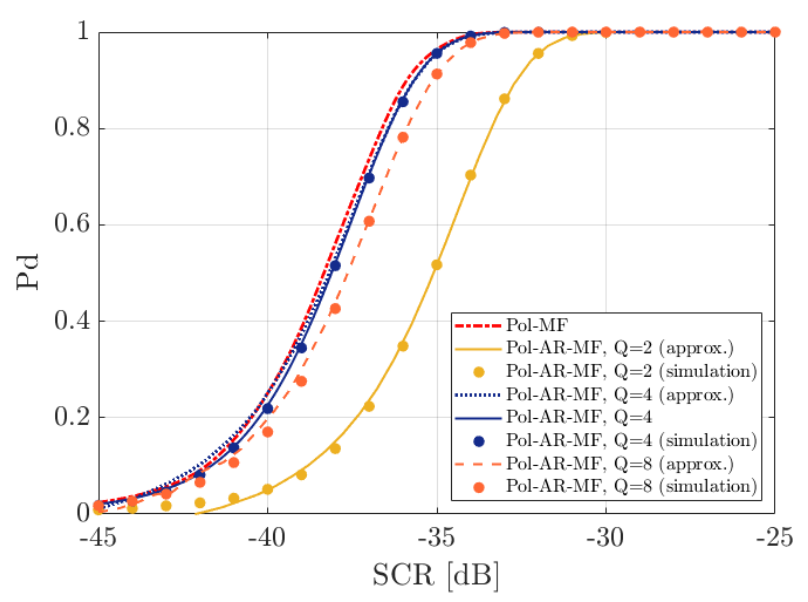

(a)

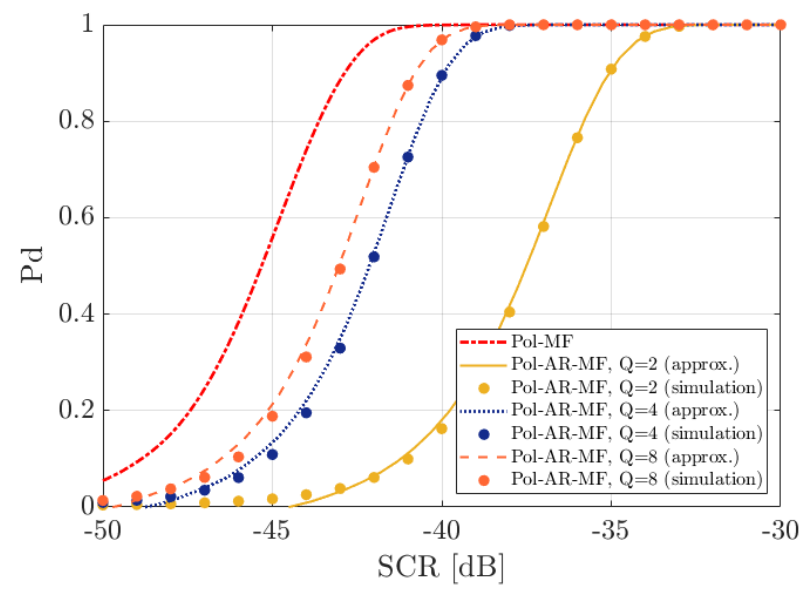

(b)

Fig. 3 Pd versus SCR for Swerling 0 target model, $P_{f a}=10^{-3}$ and (a) case A, (b) case B

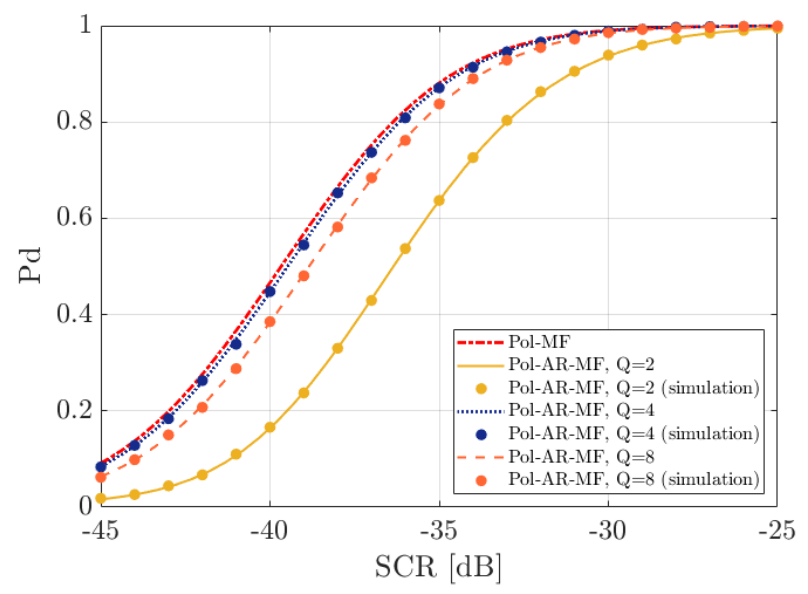

(a)

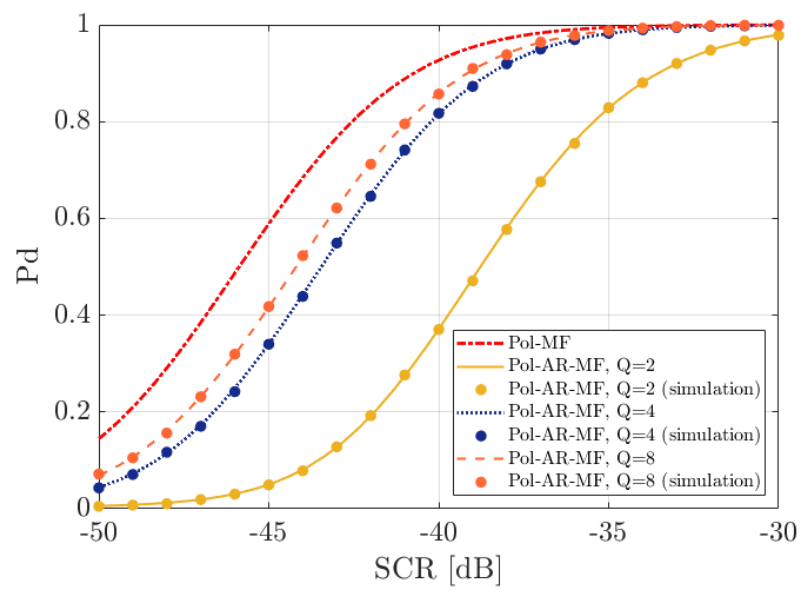

(b)

Fig. 4 Pd versus SCR for Swerling I target model, $P_{f a}=10^{-3}$ and (a) case A, (b) case B

in [1] (see eq. (31)) is reported, in solid dark blue line, for comparison. This additional comparison further confirms that (8) effectively approximates the closed-form solution when available, up to low $P_{d}$ values.

- As for Fig. 2, the better the employed AR model approximates the true spectral characteristics of the disturbance, the better the target detection performance is.

- As expected, when the case study A is considered (Fig. 3(a) and Fig. 4(a)), the 'matched' case of $Q=4$ is the best performing. However, if a limited order mismatch occurs the resulting loss is still acceptable, especially when overestimating the order of the AR process (see the curves for $Q=8$ ). In contrast, a larger performance degradation is observed when underestimating the value of $Q$ to be adopted. In the case under exam, the highest mismatch loss is obtained for $Q=2$ and it is about $3 \mathrm{~dB}$ with respect to the Pol-MF for both the non-fluctuating and fluctuating target models.

- When the case study B is considered (Fig. 3(b) and Fig. 4(b)), the detection performance of the clairvoyant Pol-AR-MF detector improves as $Q$ increases. Note that as for the $P_{f a}$, depending on the temporal correlation properties of the disturbance, the $Q$ value required to obtain a good whitening and, subsequently, a limited target detection loss with respect to the Pol-MF, might significantly change. In the case under exam, a loss smaller than $3 \mathrm{~dB}$ is reached for $Q \geq 4$ for both the non-fluctuating and fluctuating target model.

Although the considerations devised for Fig. 2, Fig. 3 and Fig. 4 specifically refer to the considered case studies, similar comments apply to alternative cases obtained with a different choice of the relevant parameters. For instance, with reference to case study B, depending on the position of employed Doppler frequency value with respect to the filter temporal notch, the behavior might not always be regular with respect to $Q$ as the filter sidelobes might not be negligible. However, we expect that a $Q$ value that better fits the data exist and that this value grows as the temporal correlation of the disturbance grows and vice versa. For instance, Fig. 5(a-b) shows the results obtained for case study B with the same parameters used in Fig. 2(b) and Fig. 4(b) but with a lower one-lag correlation coefficient value, i.e. $\varrho=0.93$. In Fig. 5 (a), we plot the $P_{f a}$ versus threshold for a grid of $Q$ values while in Fig. 5 (b) we plot the $P_{d}$ versus SCR for a Swerling I target 


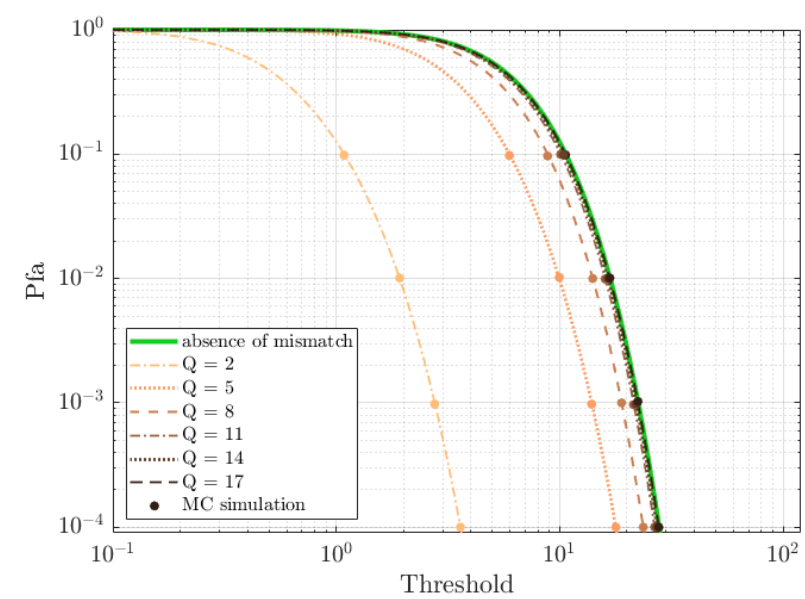

(a)

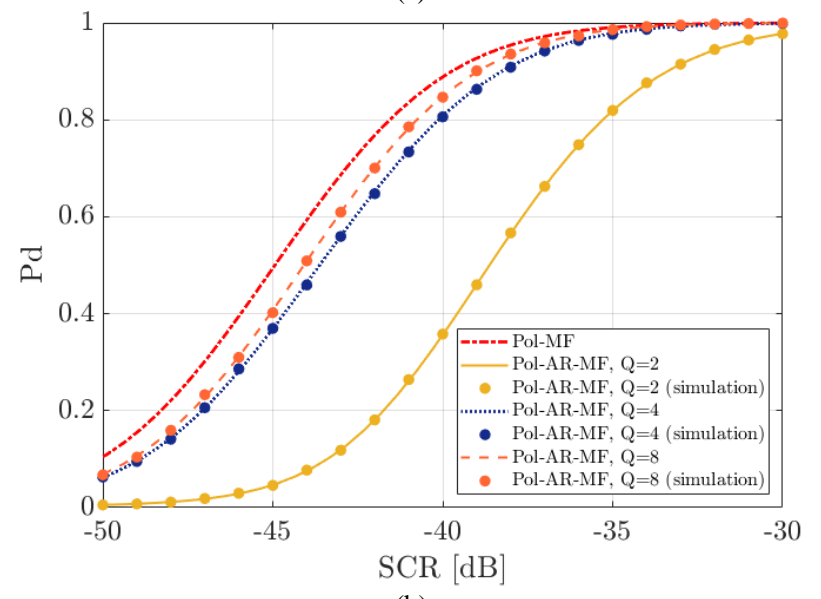

(b)

Fig. 5 Performance evaluation for case study B with $\varrho=0.93$ at $f_{d}=$ 0.28: (a) $P_{f a}$ versus threshold for different values of $Q$

(b) Pd versus SCR for Swerling I target model for $P_{f a}=10^{-3}$

model. By observing Fig. 5(a-b), we confirm that the higher is $Q$, the better is the approximation of the Gaussian shaped power spectral density based on a multi-channel AR model. However, as a lower temporal correlation is employed, a lower number of taps should be adopted to achieve an acceptable approximation. In fact, Fig. 5 (a) shows that $Q \geq 10$ allows a good approximation. Furthermore, Fig. 5 (b) shows that using $Q=4$, the clairvoyant Pol-AR-MF only yields approx. $1.5 \mathrm{~dB}$ loss with respect to the Pol-MF.

Summarizing, the analyses of $P_{f a}$ and $P_{d}$ of Sections II and III have clearly demonstrated that:

1) Spectral model mismatches jeopardize the CFAR property for the proposed detection scheme even under asymptotic conditions. In other words, a practical strategy for the threshold setting (or at least a suitable approximation [1]) is no longer available.

2) The presence of spectral model mismatches might also yield significant degradations in term of target detection capability. The observed asymptotic loss is contained within few $\mathrm{dBs}$ when a limited mismatch is present. However this might jeopardize the benefits of the proposed AR model based approaches in practical cases where a spectral mismatch typically exists and the observed loss should be summed up with the adaptivity loss deriving from an operation with a limited number $P$ of secondary data.

\section{MODIFIED AR-BASED POLARIMETRIC ADAPTIVE MATCHED FILTER}

We aim at identifying a proper modification to the proposed AR model based polarimetric detection scheme to make it robust to limited mismatches in the spectral characteristics of the disturbance. Most importantly, a practical strategy should be devised for the threshold setting in order to control the false alarm rate.

Notice that this is typically the case when no a priori information is available on the disturbance affecting the received data as in real-world radar systems. Moreover, despite effective approaches could be exploited to identify a suitable AR model that approximates the actual disturbance characteristics [5], the resulting approximation might not be perfect thus resulting in a residual spectral model mismatch.

The theoretical developments in Section II clearly show that the considered mismatch is encoded in the covariance matrix $\mathbf{D}_{0}$ of the output random vector $\breve{\mathbf{z}}_{0}$, namely the vector collecting the data after both polarimetric and temporal disturbance cancellation, which then undergoes the noncoherent integration across the polarimetric channels. This matrix tends to $2 \mathbf{I}_{L}$ if the AR model adopted for the detector design matches or very well approximates the actual disturbance characteristics. In contrast, in the presence of spectral mismatches, $\mathbf{D}_{0}$ takes alternative forms that are unknown in practice.

Therefore, an asymptotically CFAR detection scheme can be obtained by cascading an additional whitening stage with the main stages of the proposed detector aiming at restoring a polarimetrically white output $\breve{\mathbf{z}}_{0}$. Accordingly, the modified clairvoyant detector becomes:

$$
T_{\text {Pol-AR-MF }}^{\prime}=2 \mathbf{x}_{0}^{H} \mathbf{B} \mathbf{C} \mathbf{D}_{0}^{-1} \mathbf{C}^{H} \mathbf{B}^{H} \mathbf{x}_{0}{\stackrel{H_{1}}{\gtrless} \eta^{\prime}{ }_{A R-M F}}_{H_{0}}
$$

where the scaling factor 2 allows a direct comparison with the clairvoyant detector in [1]. In the following, the detection scheme in (12) will be referred to as the modified polarimetric AR model based matched filter (Mod-Pol-AR-MF).

It is easy to verify that the distribution of the detector in (12) coincides with that obtained in the 'matched' case considered in the companion paper if appropriate modifications are applied to the relevant parameters. Specifically, under the $H_{0}$ hypothesis, we might write $T^{\prime}{ }_{P o l-A R-M F}=\left\|\breve{\mathbf{z}}_{0 w}\right\|^{2}$, where $\breve{\mathbf{z}}_{0 w}$ is the whitened vector $\breve{\mathbf{z}}_{0 w}=\sqrt{2}\left(\mathbf{D}_{0}^{-1 / 2}\right)^{H} \breve{\mathbf{z}}_{0}$ and $\breve{\mathbf{z}}_{0 w} \sim \mathcal{C N}\left(\mathbf{0}_{L \times 1}, 2 \mathbf{I}_{L}\right)$. Therefore, the distribution of the test statistic is a central Chi-squared distribution with $2 L$ degrees of freedom, i.e. $T^{\prime}{ }_{P o l-A R-M F} \sim \chi_{2 L}^{2}(0)$, and the $P_{f a}$ is given by (2). 
Similarly, under the $H_{1}$ hypothesis, the theoretical $P_{d}$ expressions derived in [1] for both the non-fluctuating and fluctuating target models exactly describe the detection performance of the modified detector if the target components are properly modified. Specifically, eq. (31) of [1] holds if the non-centrality parameter of the noncentral Chi-squared distribution of the test statistic is modified as $\varsigma^{\prime}=$ $\left\|\sqrt{2}\left(\mathbf{D}_{0}^{-1 / 2}\right)^{H} \mathbf{C}^{H} \mathbf{B}^{H} \mathbf{s}\right\|^{2}$. On the other hand, eq. (32) of [1] applies if $\lambda_{0}, \ldots, \lambda_{N-1}$ denote the $N$ distinct eigenvalues of the modified covariance matrix $\mathbf{D}_{0}^{\prime \prime}=2\left(\mathbf{D}_{0}^{-1 / 2}\right)^{H} \mathbf{D}_{0}^{\prime} \mathbf{D}_{0}^{-1 / 2}$ where $\mathbf{D}_{0}^{\prime}$ was defined in Section II-B, which yields $\mathbf{D}_{0}^{\prime \prime}=$ $2 \mathbf{I}_{L}+2\left(\mathbf{D}_{0}^{-1 / 2}\right)^{H} \mathbf{C}^{H} \mathbf{B}^{H}\left(\mathbf{t t}^{H} \otimes \mathbf{M}_{\mathbf{t}}\right) \mathbf{B C} \mathbf{D}_{0}^{-1 / 2}$. These modifications basically encode the effect of the additional cancellation stage on the target and clutter components and must be carefully analyzed in order to understand whether the modified detection scheme is able to limit the target detection loss due to disturbance spectral model mismatches.

To make the detection scheme in (12) adaptive, we assume that a set of $P$ secondary data is available, $\mathbf{x}_{p}, p=1, \ldots, P$ which are target-free, independent and identically distributed (i.i.d) and share the same statistic of $\mathbf{x}_{0}$ under the $H_{0}$ hypothesis. As for the adaptive detector presented in [1], these data are first exploited to replace the unknown, though mismatched, parameters $\mathbf{A}_{m i s}$ and $\mathbf{R}_{m i s}$ within $\mathbf{C}$ and $\mathbf{B}$ with their ML estimates $\widehat{\mathbf{A}}_{\text {mis }}$ and $\widehat{\mathbf{R}}_{\text {mis }}$, obtained from the $P$ training data. Then the test statistic of the modified adaptive detector is built as:

$$
\begin{gathered}
T_{P o l-A R-A M F}^{\prime}=2 \mathbf{x}_{0}^{H} \widehat{\mathbf{B}} \hat{\mathbf{C}} \widehat{\mathbf{D}}_{0}^{-1} \widehat{\mathbf{C}}^{H} \widehat{\mathbf{B}}^{H} \mathbf{x}_{0} \underset{1}{\gtrless} \eta^{\prime}{ }_{A R-A M F} \\
H_{0}
\end{gathered}
$$

where the output covariance matrix $\mathbf{D}_{0}$ is also estimated from the secondary data once they underwent the same filtering stages applied to the primary data. In particular, according to this doubly adaptive detection scheme, we define the output of the first adaptive stage for the $p$-th input vector as $\boldsymbol{\zeta}_{p}=\widehat{\mathbf{C}}^{H} \widehat{\mathbf{B}}^{H} \mathbf{x}_{p}$, and we build an estimate of $\mathbf{D}_{0}$ as $\widehat{\mathbf{D}}_{0}=$ $\frac{1}{P} \sum_{p=1}^{P} \boldsymbol{\zeta}_{p} \boldsymbol{\zeta}_{p}^{H}$. We observe that the secondary data to be exploited for the estimation of matrix $\mathbf{D}_{0}$ should not necessarily coincide with the secondary data exploited to build the first cancellation stage of the detector.

Notice that, when $L=1$, the adaptive transformation obtained by using the inverse of matrix $\widehat{\mathbf{D}}_{0}$ would simply correspond to the scalar scaling factor of a Cell-Average CFAR autogate. In fact, we would have $\widehat{\mathbf{D}}_{0}^{-1}=\varpi^{-1}$, where $\varpi$ represents an estimate of the residual clutter power. This is obtained using $\mathrm{P}$ training data that underwent the filtering stages based on matrices $\widehat{\mathbf{B}}$ and $\widehat{\mathbf{C}}$, i.e. $\varpi=\frac{1}{P} \sum_{p=1}^{P}\left|\zeta_{p}\right|^{2}$ with $\zeta_{p}=\widehat{\mathbf{C}}^{H} \widehat{\mathbf{B}}^{H} \mathbf{x}_{p}(p=1, \ldots, P)$.

When $L>1$, the adaptive transformation obtained by using the inverse of matrix $\widehat{\mathbf{D}}_{0}$ corresponds to an additional filtering stage based on the polarimetric information extracted at the output of the previous filtering stages.
The introduced whitening stage is expected to make the false alarm control capability more robust against residual disturbance contributions that endure the first cancellation stage, as discussed in the following.

Under asymptotic conditions, $\widehat{\mathbf{A}}_{m i s}$ and $\widehat{\mathbf{R}}_{m i s}$ are asymptotic, though mismatched, estimates obtained from an infinite number of secondary data [15]. Consequently, the distribution of the test statistic of the adaptive detector in (13) tends towards that of the clairvoyant detector in (12) based on mismatched parameters.

Therefore, when exploiting a large number of training data, the performance of the modified polarimetric AR model based adaptive matched filter (Mod-Pol-AR-AMF) can be approximated by its asymptotic performance, as detailed above. Note that the CFAR property is restored for the modified adaptive detector at least in the asymptotic regime.

In this regard, we have shown in [1] that a number $P$ of training data equal to $Q L$ is typically enough to guarantee the asymptotic condition for the Pol-AR-AMF when $M$ is sufficiently high since the estimation of parameters $\widehat{\mathbf{A}}_{\text {mis }}$ and $\widehat{\mathbf{R}}_{\text {mis }}$ benefits from the average performed both across the secondary data and the temporal observations within the CPI.

However, this consideration does not apply to the additional stage included in the Mod-Pol-AR-AMF proposed in this section. In fact, this stage involves the estimation and inversion of a $L \times L$ matrix $\widehat{\mathbf{D}}_{0}$, based on the available $P$ training data. Therefore, an additional adaptivity loss is expected when operating with finite $P$, and this might result in a limited control of the $P_{f a}$ and degradations of the target detection capability.

To overcome the first issue, the fluctuations in the estimation of matrix $\widehat{\mathbf{D}}_{0}$ can be taken into account in the resulting $P_{f a}$ expression. To this purpose we make the simplified assumption that the first adaptive cancellation stage meets the asymptotic condition, namely the outputs $\boldsymbol{\zeta}_{p}$ of this adaptive stage has the same distribution of the output $\breve{\mathbf{z}}_{p}=$ $\mathbf{C}^{H} \mathbf{B}^{H} \mathbf{x}_{p}$ of the clairvoyant filter. Specifically, under the $H_{0}$ hypothesis, we have $\boldsymbol{\zeta}_{p} \sim \mathcal{C N} \mathcal{N}\left(\mathbf{0}_{L \times 1}, \mathbf{D}_{0}\right)$, being $\boldsymbol{\zeta}_{p}, p=1, \ldots$, $P$, a set of statistically independent vectors.

Under such simplified assumptions, the probability density function of the test statistic of the adaptive detector in (13), $T^{\prime}{ }_{P o l-A R-A M F}=2 \boldsymbol{\zeta}_{0}^{H} \widehat{\mathbf{D}}_{0}^{-1} \boldsymbol{\zeta}_{0}$, is well known in the technical literature for multivariate analysis as the central $F$-distribution [5]. Specifically, we have $\frac{P-L+1}{2 L P} T^{\prime}{ }_{P o l-A R-A M F} \sim F(2 L, 2(P-L+1))$ and a better approximation of the $P_{f a}$ can be obtained accordingly:

$$
P_{f a}=\frac{(1-\kappa)^{P-L+1}}{\Gamma(P-L+1)} \sum_{l=0}^{L-1} \frac{\Gamma(P-l)}{\Gamma(L-l)} \kappa^{L-l+1}
$$

being $\eta=2 P \frac{\kappa}{(1-\kappa)}$ the detection threshold.

The capability to control the false alarm rate based on (14) will be investigated in the following against both numerical and real data aiming at understanding the reliability of the adopted assumptions in practical cases. 
In turn, such analysis will also prove the CFAR property of the Mod-Pol-AR-AMF even when operating with finite $P$.

In addition, it is expected that the additional adaptive stage included in the Mod-Pol-AR-AMF might yield a nonnegligible effect on its target detection capability. The benefits/drawbacks of this effect will be studied in the following Sections both through numerical and experimental data analysis.

\section{NUMERICAL RESULTS}

First, we investigate the false alarm rate control provided by the Mod-Pol-AR-AMF when using (14) for threshold setting. Specifically, we plot in Fig. 6 the measured $P_{f a}$ versus the nominal $P_{f a}$ for case study A and different values of $Q$, namely $Q=2,4,8$ and 12 , using different training data size, namely $P=4,16$ and 192 .

We recall that (14) represents an approximated expression of the $P_{f a}$ since it relies on perfect estimates of the matrices exploited for the first adaptive stage whereas it only accounts for the fluctuations in the second adaptive stage.

Fig. 6 shows that such simplified approach allows a good control of the actual $P_{f a}$ up to reasonably low false alarm rates, at least when the number $P$ of secondary data is sufficient to provide approximate asymptotic conditions at the first adaptive stage. In this regard, we observe that some degradations appear when operating with $Q=12$ and a limited training data size. Below this value, the capability of controlling the $P_{f a}$ is ensured both when the spectral model mismatch occurs and when it does not (i.e. $Q=4$ ).

For the performance evaluation under the $H_{1}$ hypothesis, we consider the same Swerling I target model described in Section III and we plot the results in Fig. 7(a-b) for case studies A and B, respectively. Specifically, we report the target detection probability versus SCR for the Mod-Pol-ARAMF with $Q=5$ and $P=16$ or 192 .

Notice that, with the adopted value for the number $Q$ of taps, the detection scheme operates under spectral mismatched conditions in both considered case studies. In each sub-figure, we use the Pol-MF as a benchmark for the performance evaluation while the performance obtained with the adaptive Pol-GLRT [3], working with $P=2 M L=192$ secondary data, are also reported for comparison.

As it is apparent from Fig. 7 (a-b), the Mod-Pol-AR-AMF with $Q=5$ outperforms the Pol-GLRT when operating with the same number of secondary data. In fact, with $P=192$, the proposed detector approximates its asymptotic version and provides performance that is largely comparable to the ideal Pol-MF. The loss with respect to the benchmark is negligible in case study A (Fig. 7 (a)) where a limited mismatch exists between the actual spectral characteristics of the disturbance and the model adopted by the detection scheme. In contrast, a slightly higher loss is observed in case study B (Fig. 7(b)) since the detector is attempting to approximate a Gaussian spectral shaped disturbance with a multi-channel AR process of order $Q-1=4$.
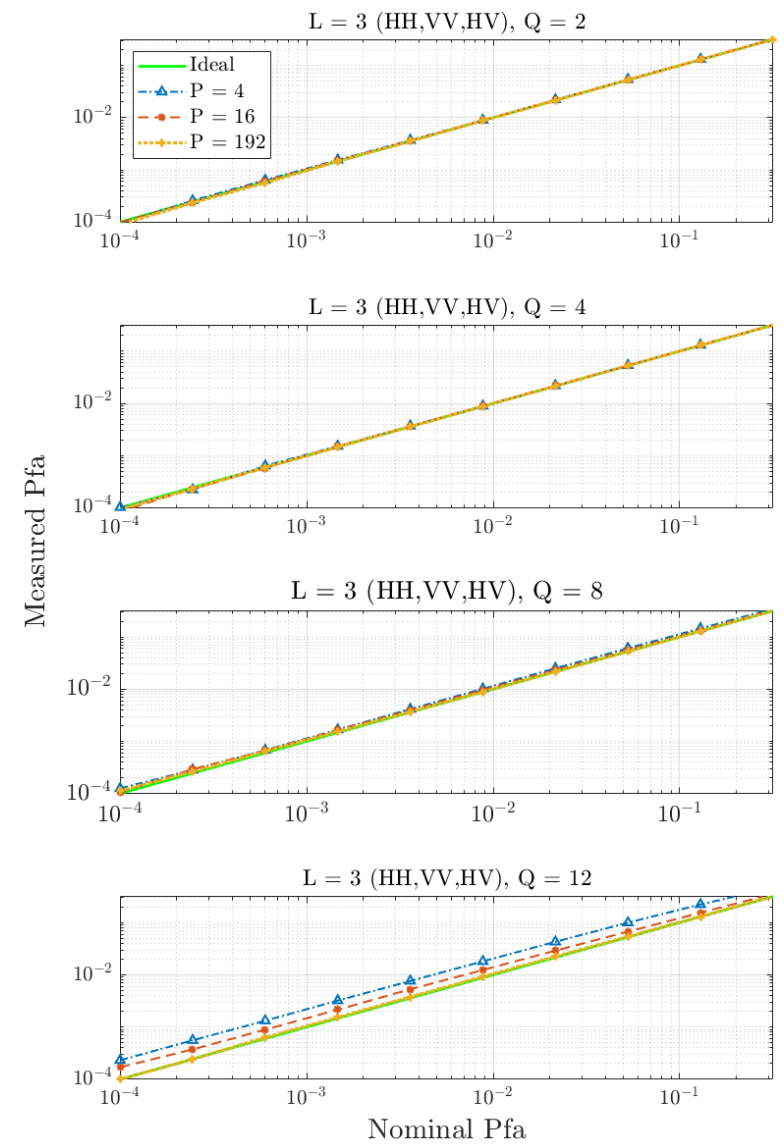

Fig. 6 Measured $P_{f a}$ versus nominal $P_{f a}$ for $M=32, L=3(\mathrm{HH}, \mathrm{VV}$, HV), different $Q$ values and different training data size.

Nevertheless, the resulting loss is smaller than $1 \mathrm{~dB}$ in the considered case study. When significantly reducing the number of secondary data, a consistent degradation is obtained with the Mod-Pol-AR-AMF which is mostly attributed to the fluctuations in the estimation of matrix $\widehat{\mathbf{D}}_{0}$, namely the second adaptive stage. In fact, following the results of [1], $P=16$ is expected to provide accurate estimates of the matrices required at the first adaptive stage when operating with $Q=5$ and $M=32$.

Anyway, thanks to the limited adaptivity loss, which is the typical benefit of parametric approaches [5]-[11] the ModPol-AR-AMF is still able to guarantee better performance with respect to the Pol-GLRT operating with a much larger training set in both the considered case studies. We incidentally observe that this result is obtained with a significantly reduced computational effort.

To complete the analysis, in Fig. 8(a-b) we compare different polarimetric detectors in terms of SCR loss with respect to the Pol-MF, measured at $P_{d}=0.9$. The Mod-PolAR-AMF is applied with a grid of $Q$ values and different $P$ values. The comparative analysis also includes the performance of the Pol-AR-MF in (1) and the Mod-Pol-ARMF in (12), which are representative of the asymptotic performance of the original proposed detector and the newly modified detection scheme, respectively. 


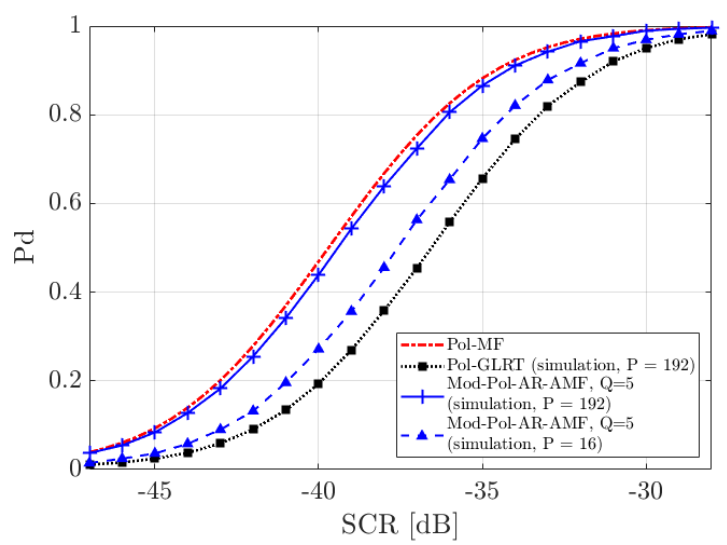

(a)

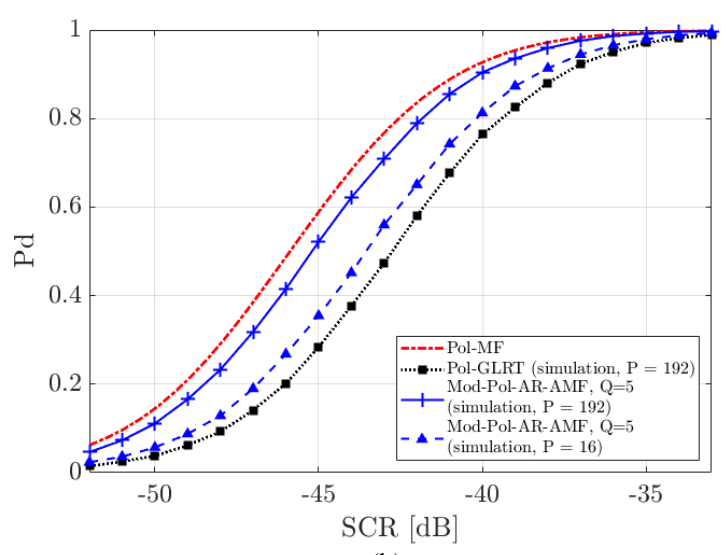

(b)

Fig. 7 Pd vs SCR for the Mod-Pol-AR-AMF against a Swerling I target model in (a) case study A, and (b) case study B.

As expected, regardless of the considered case-study, the Pol-GLRT yields a loss that only depends on the data size $L M$ and number $P$ of secondary data. This loss is equal to $3.1 \mathrm{~dB}$ and is shown as a horizontal line in Fig. 8 (a) and Fig. 8 (b).

When comparing the clairvoyant detectors, namely the Pol-AR-MF and the Mod-Pol-AR-MF, we observe that the additional cancellation stage included by the modified scheme allows to limit the spectral mismatch loss investigated in Section III. Specifically, when the detector is fed with an AR disturbance process of order $\bar{Q}-1$ (case study A, Fig. 8 (a)), the smallest asymptotic loss with respect to the Pol-MF is observed when using $Q=\bar{Q}=4$ with both detectors; we recall that this loss in this case is solely due to the border effect arising from a block-based implementation of the detection scheme as discussed in [1].

In contrast, when underestimating or overestimating the order of the AR process, the Mod-Pol-AR-MF limits the asymptotic loss thus making the resulting scheme more robust to AR model order mismatches with respect to the Pol-ARMF. Similar considerations apply to the asymptotic performance obtained in case-study B (Fig. 8 (b)) for a Gaussian spectral shaped disturbance. In this case, the asymptotic performance of the Pol-AR-MF slowly improves as the number of taps $Q$ increases. In contrast, using the modified detector a much smaller $Q$ is required to achieve negligible asymptotic loss with respect to the Pol-MF; for instance, in the considered case study B, further increasing $Q$ beyond $Q=5$, does not yield significant improvements in terms of target detection capability as the reduced spectral mismatch loss is compensated by a larger border effect when a blockbased implementation is considered for the proposed scheme.

It is then interesting to understand the effect of the additional adaptivity loss when operating with a finite number $P$ of secondary data based on the proposed Mod-Pol-ARAMF. As is apparent from Fig. 8 (a-b), the Mod-Pol-ARAMF with $P=192$ basically reaches the asymptotic performance for all the considered values of the number $Q$ of taps. Therefore, we can conclude that when operating with the same number of secondary data, the proposed Mod-Pol-ARAMF outperforms the performance of the Pol-GLRT for almost every considered value of $Q$.

When reducing the size of the training set, an additional adaptivity loss is experience by the Mod-Pol-AR-AMF (see the curves for $P=32$ and $P=16$ ). Notice that the additional loss is almost independent of the number of taps since it is mostly due to the fluctuations in the estimation of the $L \times L$ matrix $\widehat{\mathbf{D}}_{0}$ to be used in the second adaptive stage, which is the price to be paid to benefit from the observed advantages.

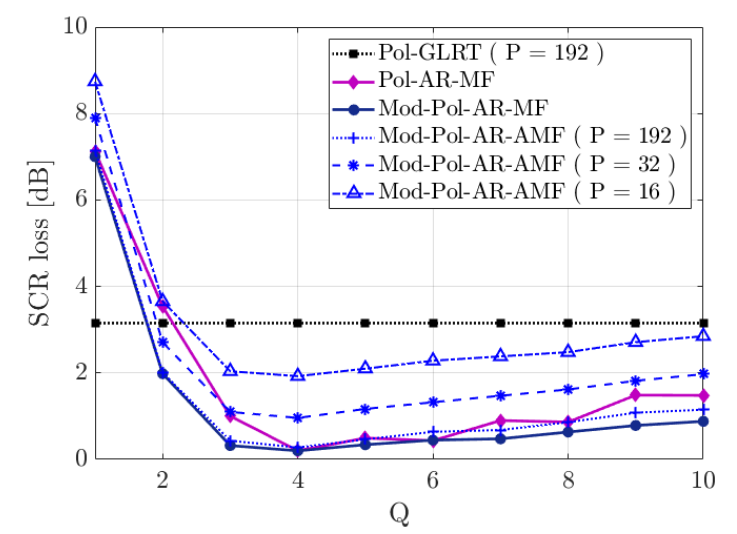

(a)

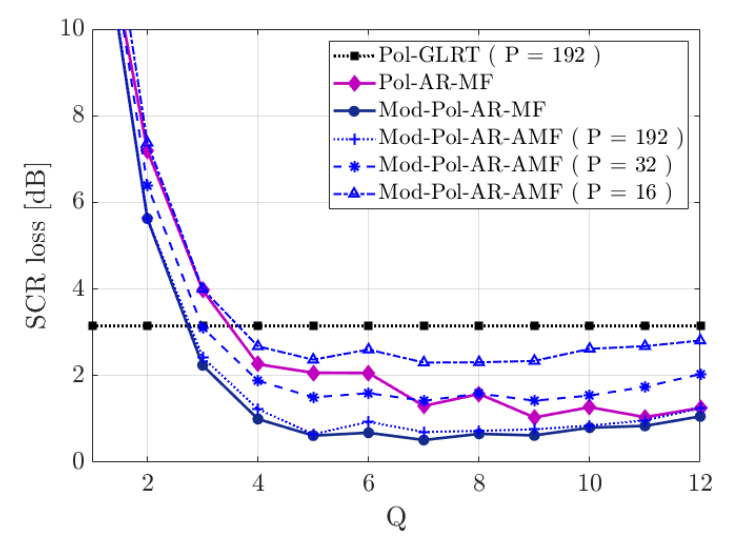

(b)

Fig. 8 SCR loss versus $Q$ for different detection schemes against a Swerling I target model in (a) case study A and (b) case study B. 
Nevertheless, in the considered case studies, the Mod-PolAR-AMF retains its performance improvement over the PolGLRT even when using a number of training data of $P=16$, namely 12 times smaller than the one used for the Pol-GLRT.

The numerical analyses reported in this section have clearly demonstrated the robustness of the proposed modified AR based polarimetric detector in both controlling the false alarm rate and reducing the detection loss under limited spectral model mismatches. These characteristics are further investigated in the following against experimental data sets.

\section{PERFORMANCE ASSESSMENT AGAINST EXPERIMENTAL DATA}

In order to prove its suitability in real world scenarios, the performance of the proposed polarimetric detection scheme are investigated in the following against two experimental data sets collected by different radar systems for diverse applications. First, we investigate the performance of the proposed Mod-Pol-AR-AMF against sea clutter measurements collected by means of an active radar. Then, we test its effectiveness against experimental data collected by means of a FM radio based passive radar system.

\section{A. Application to Active Radar Data}

The sea clutter radar measurements employed in this subsection have been collected at the Osborne Head Gunnery Range (OHGR), Dartmouth, Nova Scotia, Canada, using the McMaster IPIX radar [17]. The IPIX radar is a polarimetric radar system that alternatively transmits bursts of pulses in each of two linear polarizations $(\mathrm{H}$ and $\mathrm{V})$ and receives the corresponding back-scattered echoes at both polarizations with two parallel receiving channels. The experiment description as well as information concerning the radar parameters, the sea state, etc. are reported in [17], [18]. The sea clutter data collected my means of the IPIX radar have been widely used in the literature, see e.g. [3],[19]-[23]. Specifically, the results reported in this work refer to the target-free data file starea4 collected on November 6, 1993. The auto- and cross- spectra of the HH, VV and HV channels, averaged out over the range bins, are reported in Fig. 9 in black, blue and red, respectively.

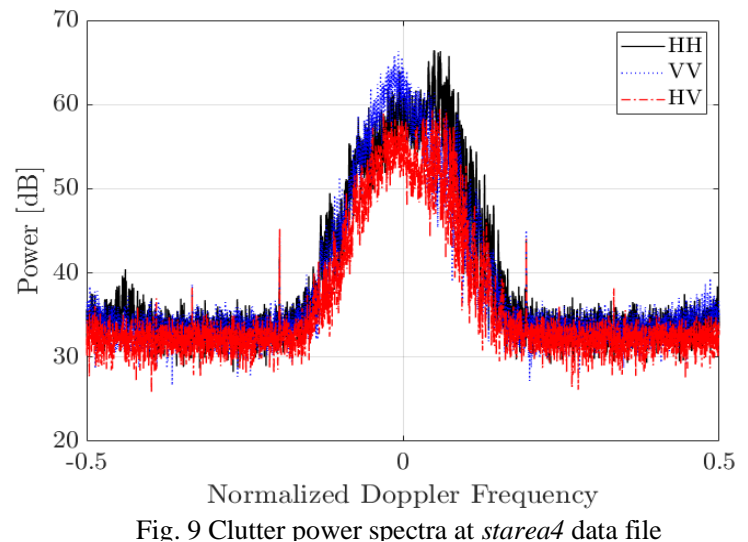

Fig. 9 Clutter power spectra at starea 4 data file
In order to preliminary investigate the capability of the Mod-Pol-AR-AMF of discriminating targets against the background, we first consider a sample data sub-set that collects the range sweeps from three polarimetric channels, i.e. $L=3(\mathrm{HH}, \mathrm{VV}, \mathrm{HV})$, at $M=32$ consecutive pulses.

Before applying the proposed detector, a fictitious pointlike target is injected at range cell 6 and $f_{d}=0.25$.As for the simulated analysis, the target complex amplitudes are set as $\boldsymbol{\alpha}=a_{t}\left[\begin{array}{lll}1 & \mathrm{e}^{j \Delta \phi_{H H / V V}} & \sqrt{\xi_{t}} \mathrm{e}^{j \Delta \phi_{H H / H V}}\end{array}\right]^{T}$, with $a_{t}$ selected to guarantee SCR at the first polarimetric channel equal to SCR $=-25 \mathrm{~dB}, \xi_{t}=10, \Delta \phi_{H H / V V}=\pi / 4$, and $\Delta \phi_{H H / H V}=\pi / 2$.

We report in Fig. 10(a-d) the normalized range-Doppler maps, resulting after applying the Mod-Pol-AR-AMF across the available range cell with different $Q$ values, namely $Q=1,2$, 4 and 8. Fig. 10 shows that, when using Mod-Pol-AR-AMF with $Q=1$ (see Fig. 10 (a)), the clutter contribution at the considered CPI is such that the target is very unlikely to be discriminated. Then, as $Q$ increases, the disturbance level is progressively reduced, and the target peak becomes more evident thus it could be easily distinguished from the background. The performance is comparable from $Q=2$ to $Q$ $=5$, although we do not show all the cases here for brevity. Afterwards, as the number of taps further increases, the background level starts slowly increasing again and Fig. 10(d) shows an example for $Q=8$. Similar results are obtained when considering different CPIs within the available data. Therefore, in the following, we focus our analysis on a limited set of choices for the number of taps $Q$, which also represent suitable values for practical applications.

For a complete performance assessment, we carried out an extensive analysis by evaluating the false alarm rate control capability as well as the target detection performance over the entire data set. In Fig. 11, we plot the measured $P_{f a}$ versus the nominal $P_{f a}$ for three values of $Q$, namely $Q=3,4$ and 5 and two different numbers of training data, namely $P=192$ and 16. In each sub-plot, we show two different results. In magenta, we plot the results obtained when using the original proposed detector Pol-AR-AMF by setting the detection threshold according to (2), namely by assuming that (i) a perfect matching exists between the spectral model adopted to

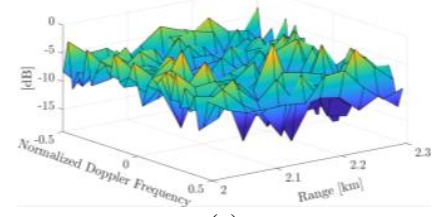

(a)

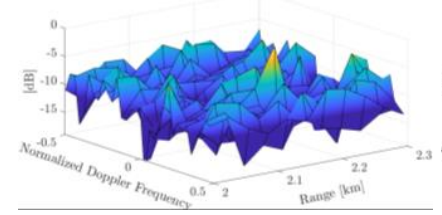

(c)

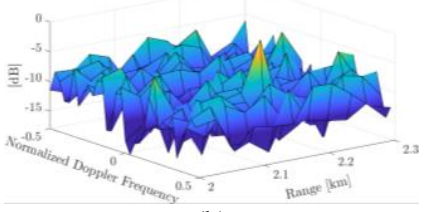

(b)

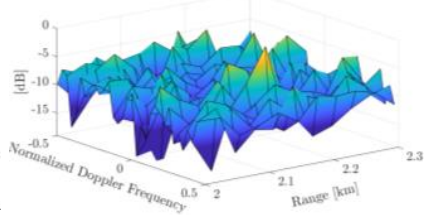

(d)
Fig. 10 Range - Doppler maps after using the Mod-Pol-AR-AMF with $P=32$, and (a) $Q=1$ (b) $Q=2$ (c) $Q=4$ (d) $Q=8$. 
L $3(\mathrm{HH}, \mathrm{VV}, \mathrm{HV}) \mathrm{Q}=3$

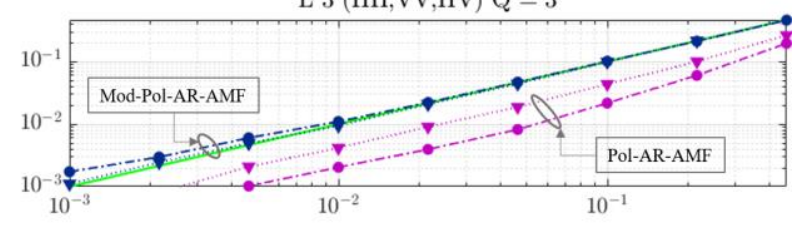

L $3(\mathrm{HH}, \mathrm{VV}, \mathrm{HV}) \mathrm{Q}=4$

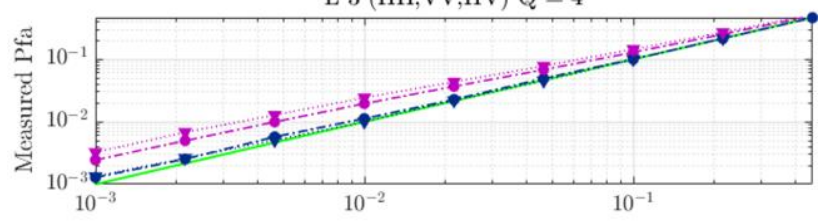

L $3(\mathrm{HH}, \mathrm{VV}, \mathrm{HV}) \mathrm{Q}=5$

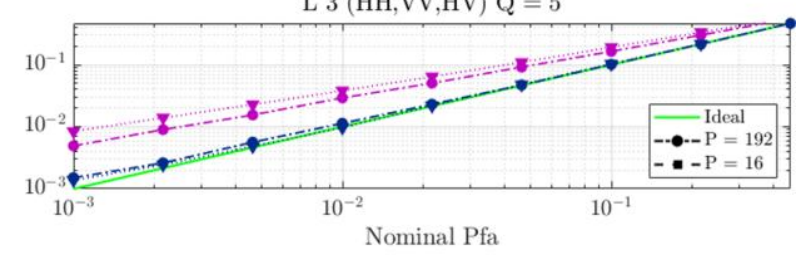

Fig. 11 Measured $P_{f a}$ versus nominal $P_{f a}$ for $M=32$,

$L=3$ (HH, VV,HV), different $Q$ values and different training data

build the detector and the actual disturbance characteristics and (ii) the Pol-AR-AMF is working in the asymptotic regime, where the employed $P_{f a}$ expression is valid. Fig. 11 clearly shows that the latter assumptions are too strict and that (2) would not provide an acceptable capability of controlling the false alarm rate for any value of $P$.

However, these results also show that, among the set of $Q$ values considered in Fig. 11, $Q=4$ appears to be the one that yields a better false alarm rate control based on the above strategy. In turn, this suggests that an AR(3) model provides a reasonable approximation of the spectral characteristics of the data at this data file [19]. In the same figures, the dark blue lines show the results obtained with the Mod-Pol-AR-AMF, when selecting the detection threshold according to (14). Fig. 11 confirms that the modified detector is robust to both spectral mismatches and non-asymptotic conditions since a quite good control of the $P_{f a}$ is guaranteed for all considered combinations of values for $Q$ and $P$ up to $P_{f a}=10^{-3}$. Incidentally, we note that lower $P_{f a}$ values could not be estimated due to the limited size of the considered data set.

Afterwards, the $P_{d}$ was investigated by injecting a fictitious Swerling I target into the sea clutter measurements before applying the proposed Mod-Pol-AR-AMF. The same parameters adopted in Section III have been used for generating the target amplitudes across the polarimetric channels. We plot the results in Fig. 12 for $P_{f a}=10^{-3}$ and $Q=$ 4 , using different numbers of polarimetric channels, i.e. $L=1$ (HH), $L=2$ ( HH, VV), $L=3$ ( HH, VV,HV), and different amount of secondary data. For comparison, we also report the performance of the Pol-GLRT operated using $P=2 M L$ in dotted black. By observing Fig. 12, we notice that as the number of available polarimetric channels increases, the target discrimination capability increases and the performance of all

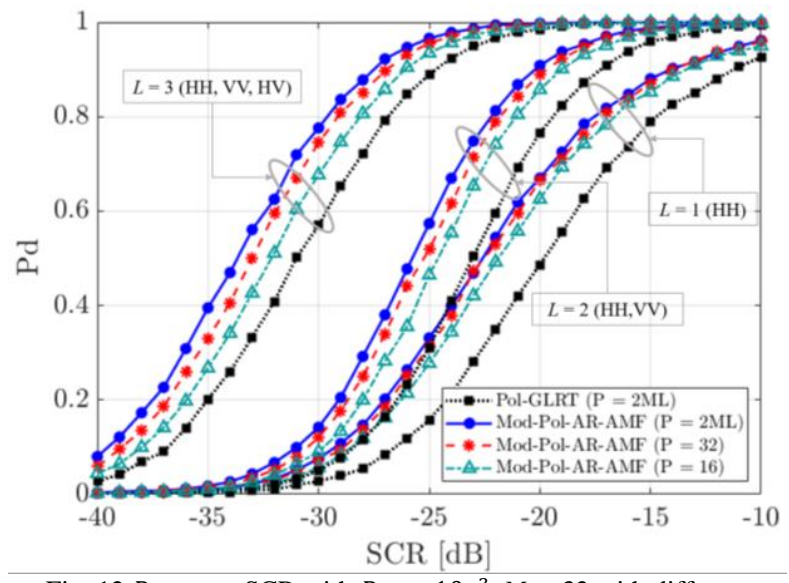

Fig. $12 P_{d}$ versus SCR with $P_{f a}=10^{-3}, M=32$ with different polarimetric channels for $Q=4$

polarimetric adaptive detectors improves. In particular, due to the higher SCR at the cross-polarized channel, using $L=3$ $(\mathrm{HH}, \mathrm{VV}, \mathrm{HV})$ yields a considerable performance improvement. When comparing the Mod-Pol-AR-AMF and the Pol-GLRT, the results in Fig. 12 largely confirms the simulated analyses. In fact, we observe that, using the same number of training data $(P=2 M L)$, the Mod-Pol-AR-AMF remarkably outperforms the Pol-GLRT. This consideration applies even when using much fewer training data $(P=32$ or 16), especially when $L=1$ or $L=2$ polarimetric channels are considered. As expected, the advantage of the Mod-Pol-ARAMF over the Pol-GLRT slightly reduces when $L=3$; however, it can be still regarded as the most suitable approach against the considered data set.

Finally, in Fig. 13 we study the detection performance as a function of the Doppler frequency and the target polarimetric cross-correlation coefficient $\rho_{t}$. Specifically, we consider $P_{f a}=10^{-3}$ and we inject a fictitious point like target using the same model adopted for Fig. 12 with SCR $=-20 \mathrm{~dB}$. In Fig. 13 (a), we report the $P_{d}$ obtained with the proposed ModPol-AR-AMF as a function of $\rho_{t}$ and $f_{d}$ with $P=2 M L=128$. In Fig. 13 (b) we focus on the extreme values of $\rho_{t}$ and we compare the Mod-Pol-AR-AMF with $P=128$ against the PolGLRT using the same training data and the Mod-Pol-ARAMF operated using $P=16$. Specifically, continuous lines refer to the case $\rho_{t}=0$ while dashed lines are for $\rho_{t}=0.99$. In both subfigures, a logarithmic scale has been used to enhance the difference at high $P_{d}$ values. Fig. 13 (a-b) confirms that the lower is the target polarimetric correlation, the narrower is the cancellation notch resulting from the Mod-Pol-AR-AMF. This result is well in line with the simulated analysis reported in [1], however here the difference between the case of $\rho_{t}=$ 0 and $\rho_{t}=0.99$ is slightly reduced, revealing a lower polarimetric correlation for the clutter [3].

The same behavior is obtained using the Pol-GLRT, however Fig. 13(b) shows that the proposed target detector outperforms the Pol-GLRT both when using the same number of training data $(P=128)$ and when using a much lower one $(P=16)$. Incidentally, when using $P=128$, the worst result 


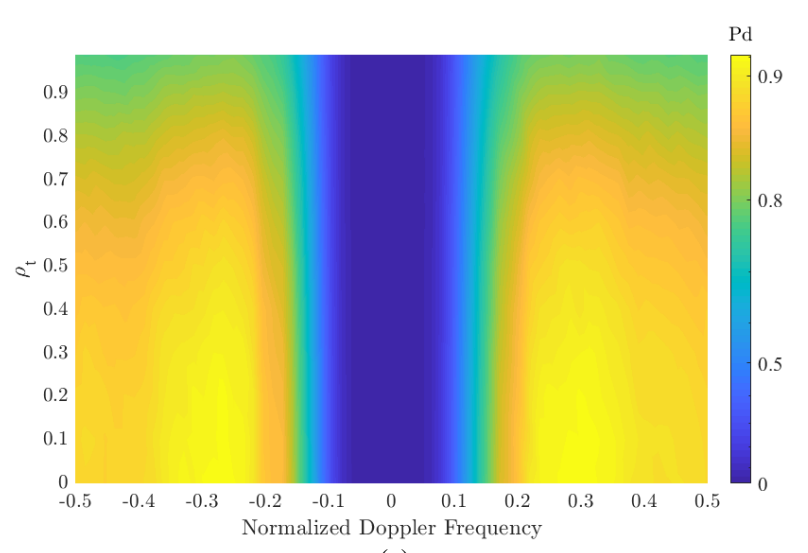

(a)

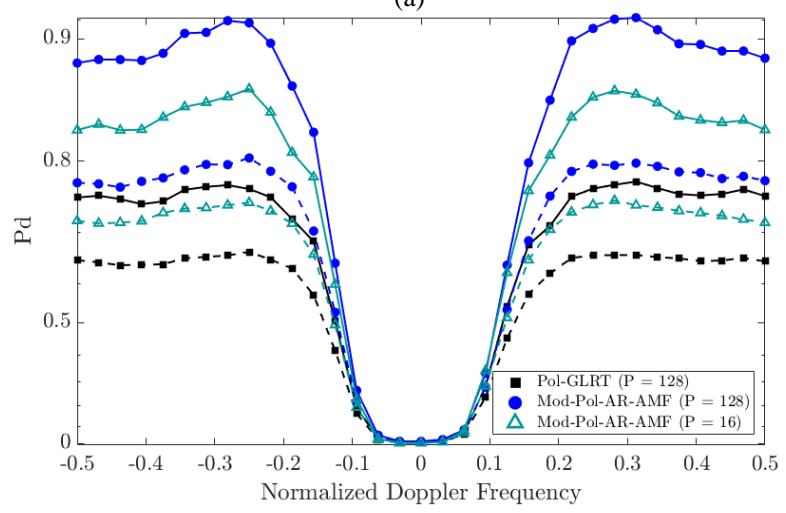

(b)

Fig. $13 P_{d}$ for $\mathrm{SCR}=-20 \mathrm{~dB}, P_{f a}=10^{-3}, L=2(\mathrm{HH}, \mathrm{VV}), M=32$ : (a) $P_{d}$ for Mod-Pol-AR-AMF $(P=128)$ as a function of $f_{d}$ and $\rho_{t}$ (b) $P_{d}$ versus $f_{d}$ for $\rho_{t}=0$ (continuous lines) and $\rho_{t}=0.99$ (dashed lines).

obtained with the Mod-Pol-AR-AMF $\left(\rho_{t}=0\right.$, see dashed blue curve) still outperforms the best result obtained with the PolGLRT $\left(\rho_{t}=0.99\right.$, see continuous black curve).

\section{B. Application to Passive Radar Data}

In the recent years, the exploitation of polarimetric diversity for target detection performance improvement has also been considered in passive radar systems [24]-[29] where the derived polarimetric adaptive detector can also find application, as preliminary shown in [29]. To this purpose, $L$ polarimetric channels could be implemented in the passive system by connecting $L$ differently polarized surveillance antennas to parallel receiving channels that simultaneously collect the corresponding signals.

In such conditions, the proposed Mod-Pol-AR-AMF can be applied with only a few adjustments that can be easily accommodated into the general signal model adopted in [1]. Specifically, in this case, the $M$ 'temporal observations' are provided by $M$ consecutive samples of the received signals included in the CPI. Correspondingly, the temporal steering vector $\mathbf{t}$ coincides with $M$ samples of the signal collected at the reference channel, which provides a good copy of the transmitted signal. Note that, in passive radar applications, long CPIs (in the order of seconds) are typically used to attain desired levels of signal-to-noise ratio (SNR). Consequently, the number of samples $M$ is usually very high (in the order of $10^{6}$ ) and this prevents the direct application of the Pol-GLRT due to (i) the unfeasible requirement of training data, and (ii) the prohibitive complexity of required computations. In contrast, suitable ML estimates of matrices $\mathbf{A}_{m i s}$ and $\mathbf{R}_{m i s}$ to be used in the Mod-Pol-AR-AMF can be obtained based upon proper signal fragments, assuming that the target contribution is negligible with respect to the competing disturbance. The estimation of matrix $\widehat{\mathbf{D}}_{0}$, is instead performed using $P=32$ secondary data surrounding the cell under test in the bistatic range-Doppler plane.

For the purpose of our analysis, we use the same data set used in [24],[26] and [29] for the case of a FM radio based passive radar system. Specifically, the acquisition campaign has been conducted near the Fiumicino Airport, Italy, exploiting a FM radio transmitter located in Monte Cavo, approx. $35 \mathrm{~km}$ from the receiver site. Two dual-polarized log periodic antennas were used as reference and surveillance antennas, being each one equipped with two independent, one vertical and one horizontal polarized, outputs. We consider the FM channel at $91.2 \mathrm{MHz}$ and we first perform a reference signal-based disturbance cancellation stage, separately at each channel, aimed at removing the direct signal and multipath contributions [21]. Then we report in Fig. 14 the results obtained with the proposed detection scheme for a single data file among the considered data set.

In particular, we show the results obtained $L=1$ and $Q=$ 1 in Fig. 14(a) and (b) for the $\mathrm{H}$ and $\mathrm{V}$ channels, respectively. In this case, no adaptive cancellation is performed either in the polarimetric and temporal domain and a conventional singlepol processing scheme is performed. The case of $L=1$ and $Q$ > 1 is reported in Fig. 14(c) and (d) for $Q=3$. Finally, the results obtained when jointly exploiting the $L=2$ polarimetric channels and adaptively rejecting the disturbance according to the proposed Mod-Pol-AR-AMF with $Q=1$ or $Q=3$ are reported in Fig. 14(e) and (f), respectively.

Specifically, for each case, we report the test statistics over the bistatic range-velocity plane before the application of a proper threshold, selected according to a desired value of nominal $P_{f a}$. For a fair comparison, the test statistic is mapped into the $P_{f a}$ setting that would allow the corresponding threshold exceeding. In other words, each pixel in the map has been scaled so that it represents the minimum value of nominal $P_{f a}$ to be set for that pixel to yield a detection.

Additionally, based on the available ATC registrations, all targets that were present at the time of the considered data file are marked with circles. In detail, we used green circles for targets that would be detected for $P_{f a} \leq 10^{-4}$ and red circles for the others.

By observing Fig. 14(a-f), the following considerations are in order:

- When the single-pol channels are separately used, and processed according to the conventional PCL processing scheme, some missed detections are obtained. Moreover, by comparing Fig. 14(a) and Fig. 14(b), most of these 


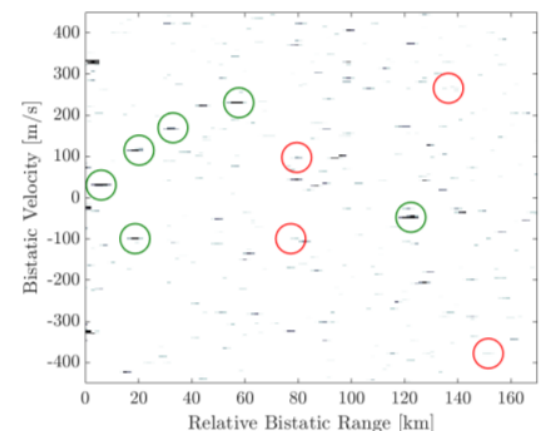

(a)

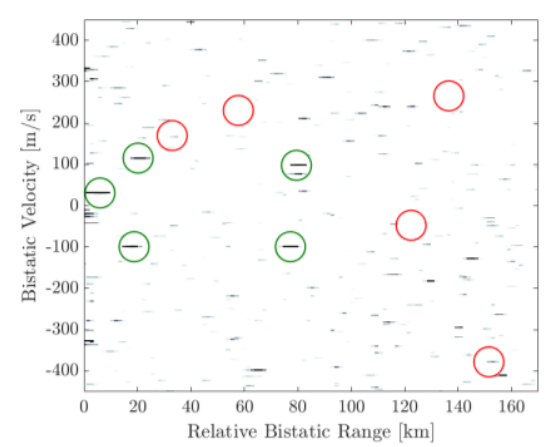

(b)

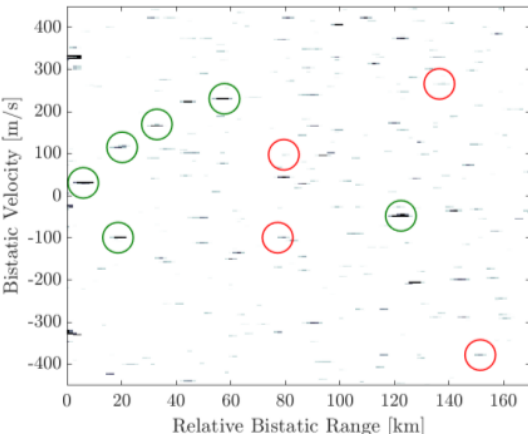

(c)

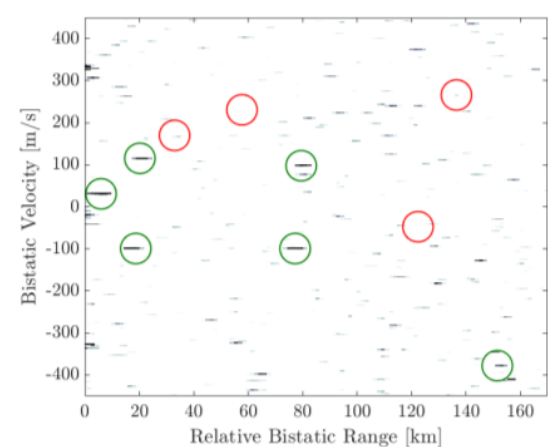

(d)

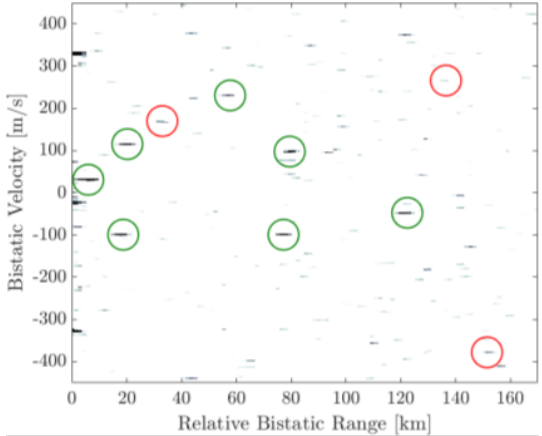

(e)

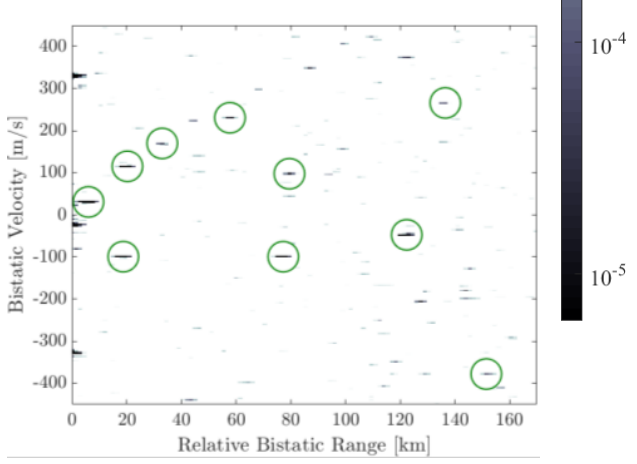

(f)

Fig. 14 Minimum $P_{f a}$ to set to detect each bin, using Mod-Pol-AR-AMF with (a) $L=1(\mathrm{H})$ and $Q=1$; (b) $L=1(\mathrm{~V})$ and $Q=1$; (c) $L=1(\mathrm{H})$ and $Q=3$; (d) $L=1(\mathrm{~V})$ and $Q=3(\mathrm{e}) L=2(\mathrm{H}, \mathrm{V})$ and $Q=1 ;(\mathrm{d}) L=2(\mathrm{H}, \mathrm{V})$ and $Q=3$.

missed detections are complementary, which confirms the information diversity conveyed by different polarimetric channels. Furthermore, the high background level would yield a non-negligible number of false alarms even at low $P_{f a}$ (see the number of isolated peaks that do not correspond to any target in Fig. 14(a) and (b)).

- When the single-pol channels are separately used but the AR model based detector is performed using $Q=3$, (see Fig. 14(c-d)), only a slight improvement is obtained with respect to the case of $Q=1$ at the $\mathrm{V}$ channel (see Fig. 14(d)). However, the number of isolated peaks that would exceed the threshold although not associated to any target, is still quite high revealing that the considered strategy is not able to effectively reject the disturbance at this data file.

- When only exploiting the polarimetric diversity (see Fig. 14(e)), a lower number of missed detections is obtained and the background level is significantly reduced. However, note that the farthest target that was recovered in Fig. 14(d) would exceed the threshold only at higher $P_{f a}$ in this case.

- Finally, when performing the proposed polarimetric adaptive detector using both the $\mathrm{H}$ and $\mathrm{V}$ channels and $\mathrm{Q}=$ 3 (see Fig. 14(f)), all targets appear as isolated peaks and would be correctly detected at $P_{f a} \leq 10^{-4}$. Moreover, the number of false alarms has been significantly lowered, confirming the capability of the devised approach to effectively counteract the disturbance thanks to the exploited information diversity.

These results show the potentiality of the proposed polarimetric adaptive detector also for passive radar systems, as also preliminary shown in [29]. Clearly, for a complete performance assessement, an extensive analysis over the entire data set must be carried out. However, despite computationally more efficient than the Pol-GLRT, the direct implementation of the proposed Mod-Pol-AR-AMF to the passive radar case still requires a high computational burden that makes the aforementioned extensive analyses unsuitable. Therefore, future works will address possible strategies that aim at reducing the computational load while accepting small losses.

\section{CONCLUSION}

In this paper, the performance of the polarimetric adaptive detector presented in the accompanying paper [1] has been extensively studied the case of an input disturbance that does not perfectly match the AR model used for the derivation of the detection test. The theoretical and simulated analyses have revealed the limitations of the original detector under such conditions and these include the loss of the asymptotic CFAR property and subsequent degradations in terms of target detection capability. Consequently, the authors introduced an appropriate modification to the devised detector to make it robust to typical spectral mismatches occurring in practical situations. Eventually, the effectiveness of the resulting detection scheme has been demonstrated against both simulated and experimental data, where the modified detector was proven to guarantee a remarkable control of the false alarm rate and target detection performance outperforming the traditional detection schemes. 


\section{APPENDIX \\ APPROXIMATION OF THE ASYMPTOTIC PD FOR NON-FLUCTUATING TARGET}

In this Appendix, we look for $\operatorname{Prob}\left\{\left\|\breve{\mathbf{z}}_{0}\right\|^{2}>\eta\right\}$, where vector $\breve{\mathbf{z}}_{0}$ is a complex Gaussian random variable with mean vector $\boldsymbol{S}$ and covariance matrix $\mathbf{D}_{0}$. To this end, we follow the main steps of the procedure reported in [16], with reference to the problem under consideration.

For the results in [16] to be applied, we first write vector $\breve{\mathbf{z}}_{0}$ as $\breve{\mathbf{z}}_{0}=\boldsymbol{\varsigma}+\boldsymbol{v}$, where $\boldsymbol{\varsigma} \sim \mathcal{C N}\left(\mathbf{0}_{L \times 1}, \mathbf{D}_{0}\right)$. Then we rewrite the test statistic as $T_{P o l-A R-M F}=\left(\boldsymbol{\varsigma}_{w}+\mathbf{v}_{w}\right)^{H} \mathbf{D}_{0}\left(\boldsymbol{\varsigma}_{w}+\mathbf{v}_{w}\right)$, denoting $\boldsymbol{\zeta}_{w}=\left(\mathbf{D}_{0}^{-1 / 2}\right)^{H} \boldsymbol{\varsigma}$ as a white complex Gaussian random vector, i.e. $\boldsymbol{S}_{w} \sim \mathcal{C N}\left(\mathbf{0}_{L \times 1}, \mathbf{I}_{L}\right)$ and $\boldsymbol{v}_{w}=\left(\mathbf{D}_{0}^{-1 / 2}\right)^{H} \boldsymbol{v}$. Moreover, let us consider the eigenvalue decomposition of matrix $\mathbf{D}_{0}$, i.e. $\mathbf{D}_{0}=\mathbf{K} \boldsymbol{\Lambda} \mathbf{K}^{H}$ and let $\lambda_{0} \ldots, \lambda_{L-1}$ denote the eigenvalues of $\mathbf{D}_{0}$.

According to this model, we look for the cumulative distribution function (CDF) of $T_{P o l-A R-M F}$ which can be written as follows [16]

$$
\begin{gathered}
F_{0}(t)=\frac{1}{2 \pi} \int_{-\infty}^{\infty} \frac{e^{t(j \omega+\beta)}}{(j \omega+\beta)} \frac{e^{-c(\omega)}}{\left|\mathbf{I}_{L}+(j \omega+\beta) \boldsymbol{\Lambda}\right|} d \omega \\
(\beta>0, t>0)
\end{gathered}
$$

with $c(\omega)=\overline{\overline{\mathbf{v}}}^{H}\left(\mathbf{I}_{L}+\frac{1}{j \omega+\beta} \boldsymbol{\Lambda}^{-1}\right)^{-1} \overline{\overline{\mathbf{v}}}, \overline{\overline{\mathbf{v}}}=\mathbf{K}^{H} \mathbf{v}_{w}$.

A closed form solution for the integral in (15) cannot be obtained in the general case. Therefore, as in [16], we derive an approximation of the sought $P_{d}$ expression by resorting to the saddle point (SP) technique [30]. To this end, we define

$$
\begin{aligned}
f(\omega)=t(j \omega+\beta) & -\ln (j \omega+\beta) \\
& +\sum_{l=0}^{L-1}\left|\overline{\bar{v}}_{l}\right|^{2}\left[\frac{1}{1+(j \omega+\beta) \lambda_{l}}-1\right] \\
& -\sum_{l=0}^{L-1} \ln \left[1+(j \omega+\beta) \lambda_{l}\right]
\end{aligned}
$$

and, consequently, we write (15) as

$$
F_{0}(t)=\frac{1}{2 \pi} \int_{-\infty}^{\infty} e^{f(\omega)} d \omega
$$

To apply the SP technique, we first differentiate $f(\omega)$ and look for the only real solution of $\dot{f}(\omega)=0$, denoted as $\omega_{0}=$ $j\left(\beta+p_{0}\right)$, in the region $p \in(-\infty, 0)$.

$$
\begin{gathered}
\dot{f}(\omega)=-\sum_{l=0}^{L-1} \frac{j \lambda_{l}}{1+(j \omega+\beta) \lambda_{l}}\left[1+\frac{\left|\overline{\bar{v}}_{l}\right|^{2}}{1+(j \omega+\beta) \lambda_{l}}\right] \\
+j t-\frac{\mathrm{j}}{(j \omega+\beta)}=0
\end{gathered}
$$

Now we approximate $f(\omega)$ using a second order Taylor expansion around $\omega_{0}$, yielding

$$
F_{0}(t) \approx \frac{e^{f\left(\omega_{0}\right)}}{\sqrt{2 \pi\left|\ddot{f}\left(\omega_{0}\right)\right|}}
$$

where $\ddot{f}\left(\omega_{0}\right)$ is derived as

$$
\begin{aligned}
& \ddot{f}\left(\omega_{0}\right) \\
& =-\left\{\frac{1}{\left(j \omega_{0}+\beta\right)^{2}}\right. \\
& \left.+\sum_{l=0}^{L-1} \frac{\lambda_{l}^{2}}{\left[1+\left(j \omega_{0}+\beta\right) \lambda_{l}\right]^{2}}\left[\frac{2\left|\overline{\bar{v}}_{l}\right|^{2}}{1+\left(j \omega_{0}+\beta\right) \lambda_{l}}+1\right]\right\}
\end{aligned}
$$

Therefore, by using (20) and by evaluating (16) in $\omega_{0}$ we obtain (21), which can be easily converted in (8).

\section{ACNOWLEDGEMENT}

The authors gratefully acknowledge the anonymous reviewers and the associate editor for their constructive comments on the manuscript.

\section{REFERENCES}

[1] F. Colone and F. Filippini: "Auto-regressive model based Polarimetric Adaptive Detection Scheme. Part I: Theoretical Derivation and Performance Analysis," accepted for pubblication in IEEE Transactions on Aerospace and Electronic Systems, COMPANION PAPER

[2] H. R. Park, J. Li, H. Wang, "Polarization-space-time domain generalized likelihood ratio detection of radar targets", Signal Processing, vol. 41, pp. 153-164, 1995.

[3] D. Pastina, P. Lombardo and T. Bucciarelli, "Adaptive polarimetric target detection with coherent radar. I. Detection against Gaussian background," in IEEE Trans. on Aerospace and Electronic Systems, 37 (4), pp. 1194-1206, Oct. 2001.

[4] A. De Maio, G. Ricci, "A polarimetric adaptive matched filter", Signal Processing, vol. 81, no. 12, pp. 2583-2589, Dec. 2001.

[5] J. J. Shynk, Probability Random Variables and Random Processes: Theory and Signal Processing Applications, New York, NY, USA:Wiley, 2012.

[6] S. Haykin, A. Steinhardt, Adaptive radar detection and estimation, New York: Wiley, 1992.

[7] J. H. Michels, P. Varshney and D. Weiner, "Multichannel signal detection involving temporal and cross-channel correlation," in IEEE Trans. on AES, vol. 31, no. 3, pp. 866-880, July 1995.

$$
\begin{gathered}
P_{d}=1-F_{0}(\eta) \approx 1-\frac{1}{\sqrt{2 \pi}}\left|-\sum_{l=0}^{L-1}\left\{\frac{2\left|\bar{v}_{l}\right|^{2} \lambda_{l}{ }^{2}}{\left[1+\left(j \omega_{0}+\beta\right) \lambda_{l}\right]^{3}}+\frac{\lambda_{l}{ }^{2}}{\left[1+\left(j \omega_{0}+\beta\right) \lambda_{l}\right]^{2}}\right\}-\frac{1}{\left(j \omega_{0}+\beta\right)^{2}}\right|^{-\frac{1}{2}} \times \exp \left\{\eta\left(j \omega_{0}+\beta\right)-\right. \\
\left.\ln \left(j \omega_{0}+\beta\right)+\sum_{l=0}^{L-1}\left|\overline{\bar{v}}_{l}\right|^{2}\left[\frac{1}{1+\left(j \omega_{0}+\beta\right) \lambda_{l}}-1\right]-\sum_{l=0}^{L-1} \ln \left[1+\left(j \omega_{0}+\beta\right) \lambda_{l}\right]\right\}
\end{gathered}
$$

(Errore.

Solo

documento principale.) 
[8] J. R. Roman, M. Rangaswamy, D. W. Davis, Qingwen Zhang, B. Himed and J. H. Michels, "Parametric adaptive matched filter for airborne radar applications," in IEEE Trans. on AES, 36, (2), pp. 677-692, Apr. 2000.

[9] K. J. Sohn, H. Li and B. Himed, "Parametric GLRT for Multichannel Adaptive Signal Detection," in IEEE Transactions on Signal Processing, vol. 55, no. 11, pp. 5351-5360, Nov. 2007.

[10] C. Hao, S. Gazor, D. Orlando, G. Foglia and J. Yang, "Parametric space-time detection and range estimation of a small target," in IET Radar, Sonar \& Navigation, vol. 9, no. 2, pp. 221-231, 22015.

[11] Y. Gao, H. Li, B. Himed, "Adaptive Subspace Tests for Multichannel Signal Detection in Auto-Regressive Disturbance," in IEEE Trans. on Signal Processing, 66 (21), pp. 5577-5587, Nov.1, 2018.

[12] H. Akaike, "A new look at the statistical model identification," in IEEE Trans. on Automatic Control, vol. 19, no. 6, pp. 716-723, Dec. 1974.

[13] P. Swerling, "Probability of detection for fluctuating targets," in IRE Trans. on Information Theory, vol. 6, no. 2, pp. 269-308, April 1960.

[14] M. I. Skolnik, Radar handbook, 3rd ed. McGraw-Hill, 2008

[15] S. M. Kay, Fundamentals of Statistical Signal Processing: Detection Theory, vol. II. Englewood Cliffs, NJ, USA: Prentice-Hall, 1993.

[16] T. Y. Al-Naffouri, M. Moinuddin, N. Ajeeb, B. Hassibi and A. L. Moustakas, "On the Distribution of Indefinite Quadratic Forms in Gaussian Random Variables," in IEEE Transactions on Communications, vol. 64, no. 1, pp. 153-165, Jan. 2016.

[17] S. Haykin, C. Krasnor, T. J. Nohara, B. W. Currie and D. Hamburger, "A coherent dual-polarized radar for studying the ocean environment," in IEEE Transactions on Geoscience and Remote Sensing, vol. 29, no. 1, pp. 189-191, Jan. 1991

[18] A. Drosopoulos, "Description of the OHGR database, " Defence Research Establishment, Ottawa, Tech. Rep. 94-14, Dec. 1994.

[19] M. Greco, F. Bordoni and F. Gini, "X-band sea-clutter nonstationarity: influence of long waves," in IEEE Journal of Oceanic Engineering, vol. 29, no. 2, pp. 269-283, April 2004.

[20] M. Greco, F. Gini and M. Rangaswamy, "Statistical analysis of measured polarimetric clutter data at different range resolutions," in IEE Proceedings - Radar, Sonar and Navigation, vol. 153, no. 6, pp. 473481, December 2006.

[21] A. Farina, F. Gini, M. V. Greco and L. Verrazzani, "High resolution sea clutter data: statistical analysis of recorded live data," in IEE Proceedings - Radar, Sonar and Navigation, vol. 144, no. 3, pp. 121130, June 1997.

[22] F. Gini, M. V. Greco, M. Diani and L. Verrazzani, "Performance analysis of two adaptive radar detectors against non-Gaussian real sea clutter data," in IEEE Trans. on AES, 36(4), pp. 1429-1439, Oct. 2000.

[23] A. De Maio, A. Farina and G. Foglia, "Target fluctuation models and their application to radar performance prediction," in IEE Proc. - Radar, Sonar and Navigation, vol. 151, no. 5, pp. 261-269, 10 Oct. 2004.

[24] F. Colone and P. Lombardo: "Polarimetric passive coherent location", IEEE Transactions on Aerospace and Electronic Systems, 2015, 51, (2), pp. 1079-1097.

[25] J. You, X. Wan, Y. Fu and G. Fang, "Experimental study of polarisation technique on multi-FM-based passive radar," in IET Radar, Sonar \& Navigation, vol. 9, no. 7, pp. 763-771, 82015.

[26] F. Colone and P. Lombardo, "Non-coherent adaptive detection in passive radar exploiting polarimetric and frequency diversity," in IET Radar, Sonar \& Navigation, vol. 10, no. 1, pp. 15-23, 12016.

[27] M. Conti, C. Moscardini, A. Capria, "Dual-polarization DVB-T passive radar: Experimental results", 2016 IEEE Radar Conference, Philadelphia, PA, 2016, pp. 1-6.

[28] I. Son and B. Yazici, "Passive polarimetrie multistatic radar for ground moving target," 2016 IEEE Radar Conference, Philadelphia, PA, 2016, pp. 1-6.

[29] F. Filippini, and F. Colone,: "Polarimetric Detection Scheme for Passive Radar based on a 2D Auto-Regressive Disturbance Model", 2019 International Radar Conference (RADAR 2019), Toulon.

[30] C. M. Bender and S. A. Orszag, Advanced Mathematical Methods for Scientists and Engineers, New York, NY, USA, McGraw-Hill, 1978.

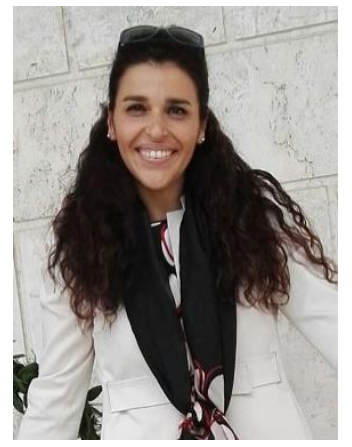

Fabiola Colone received the laurea degree (B.S.+M.S.) in Telecommunications Engineering and the Ph.D. degree in Remote Sensing from Sapienza University of Rome, Italy, in 2002 and 2006, respectively. She joined the DIET Dept. (formerly INFOCOM) of Sapienza University of Rome as a Research Associate in January 2006. From December 2006 to June 2007, she was a Visiting Scientist at the Electronic and Electrical Engineering Dept. of the University College London, London, U.K. She is currently an Associate Professor at the Faculty of Information Engineering, Informatics, and Statistics of Sapienza University of Rome.

The majority of Dr. Colone's research activity is devoted to radar systems and signal processing. She has been involved, with scientific responsibility roles, in research projects funded by the European Commission, the European Defence Agency, the Italian Space Agency, the Italian Ministry of Research, and the radar industry. Her research has been reported in over 120 publications in international technical journals, book chapters, and conference proceedings. Dr. Colone has been co-recipient of the 2018 Premium Award for Best Paper in IET Radar, Sonar \& Navigation.

Since 2017 she is member of the Board of Governors of the IEEE Aerospace and Electronic System Society (AESS) in which she is currently serving as Vice-President for Member Services, and Editor in Chief for the IEEE AESS QEB Newsletters. She is IEEE Senior Member from 2017 and member of the IEEE AESS Radar System Panel from 2019. Dr. Colone is Associate Editor for the IEEE Transactions on Signal Processing and member of the Editorial Board of the Int. Journal of Electronics and Communications (Elsevier). She served in the technical committee of many international conferences. She was in the organizing committee, as the Student Forum Co-Chair, of the IEEE 2008 Radar Conference (Rome, Italy), and she is currently in the organizing committee, as Special Sessions Co-Chair, of the IEEE 2020 Radar Conference (Florence, Italy). She is also Technical co-Chair of the IEEE Radar Conference 2021 (Atlanta, USA).

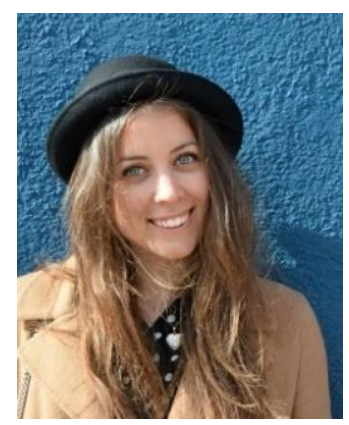

Francesca Filippini (S'17-M'20) received her M.Sc. degree (cum Laude) in Communication Engineering and the $\mathrm{PhD}$ degree in Radar and Remote Sensing, from Sapienza University of Rome, in 2016 and 2020, respectively. From January to May 2016, she has been working on her Master Thesis at Fraunhofer Institute FHR, as an intern of the Passive Radar and Anti-Jamming Techniques Department. She is currently a PostDoctoral Researcher with the DIET Dept. of Sapienza University of Rome.

Dr. Filippini received the 2018 Premium Award for the Best Paper in IET Radar, Sonar \& Navigation, the Best Paper Award at the 2019 Int. Radar Conference, the second Best Student Paper Award at the 2018 IEEE Radar Conference and the Best Paper Award at the 2017 GTTI Workshop on Radar and Remote Sensing. She is a Member of the IEEE AESS Board of Governors, where she is currently serving as Graduate Student Representative, and Chair of the AESS Professional Networking and Mentoring Program. 